\title{
A Universal Symmetry Criterion for the Design of High Performance Ferroic
}

\section{Materials}

\author{
Yipeng Gao, Suliman A. Dregia, Yunzhi Wang* \\ Watts Hall, 2041 College Rd, Department of Materials Science and Engineering, The Ohio
}

State University, Columbus, OH 43210, USA.

\begin{abstract}
:
The symmetry of a crystal has profound effects on its physical properties and so does symmetry-breaking on the characteristics of a phase transition from one crystal structure to another. For an important class of smart materials, the ferroics, their functionality and performance are associated with cycles of transitions from multiple structural states of one phase to those of the other. Using group and graph theories we construct phase transition graph (PTG) and show that both the functionality and performance of ferroics are dictated by the topology of their PTGs. In particular, we demonstrate how the giant piezoelectricity in ferroelectrics and the functional fatigue in shape memory alloys (SMAs) are related to their unique PTG topological features. Using PTG topology as a guide, we evaluate systematically new systems potentially having giant piezoelectricities and giant electro- and magneto-strictions and discuss the design strategies for high performance SMAs with much improved functional fatigue resistance.
\end{abstract}

Keywords: phase transition, crystal symmetry, transition path, ferroelectric, shape memory alloys. 


\section{Introduction}

Crystal structural changes in response to external fields (temperature, pressure/stress, electrical or magnetic, etc.) underpin the functionality of an important class of smart materials, the ferroics. Because of the symmetry-breaking associated with a structure change, there are multiple crystallographically equivalent and energetically degenerate ways to transform from one crystal structure to another (will be referred to as phase transition pathways (PTPs) hereafter), generating multiple crystallographically equivalent and energetically degenerate structural states of the product phase called transformation variants [1-4]. These variants arrange themselves into self-accommodating domain patterns and can switch from one to another by an external field, thus sensing and actuation can be realized simultaneously. The ferroics have found critical applications in many fields [5-9] and extensive efforts have been made in recent years to develop advanced ferroics with much enhanced performance, such as giant piezoelectricity, giant electro- and magneto-striction and giant super-elastic response.

It has yet to be recognized, however, that the properties of ferroics are dictated not only by the symmetry of individual crystal structures involved in and the symmetry-breaking during the phase transition, but also by the interconnection of the multiple structural states through the multiple PTPs, which yields a PTP network or phase transition graph (PTG). PTG is a new theoretical construct capturing the sequential changes of crystal structures during multiple forward and backward phase transitions (will be referred to as phase transition cycles hereafter), and it can be utilized to analyze systematically the symmetry breaking during the transition cycles as well as the associated defect structures. In the following sections, we first 
provide a rigorous mathematical definition of PTG by using group and graph theories and then demonstrate how its graph features (e.g., connectivity, topology, symmetry, etc.) dictate the properties of ferroics such as the giant piezoelectricity in ferroelectrics and functional fatigue in shape memory alloys (SMAs). In contrast to crystal physics [10] that studies the relationship between physical properties and the structure and symmetry of each individual phase, and to Landau theory [11] that studies properties of one-way structural phase transitions with symmetry-breaking, this PTG analysis studies the characteristics and physical properties of transition cycling from multiple structural states of one phase to those of the other.

In Landau theory [11] of structural phase transitions, the free energy of a low-symmetry product phase is approximated by a power series expansion with respect to the high-symmetry parent phase. However, there are two inherent deficiencies in this approach: (1) it requires a unique high-symmetry phase that has all the symmetry elements of the low symmetry phase (i.e., a group-subgroup relationship); (2) only local pathway connectivity within the vicinity of the high-symmetry phase is captured. Consider, for example, the body-centered cubic (BCC, space group $I m \overline{3} m$ ) structure to hexagonal-close-packed (HCP, space group $P 6_{3} / m m c$ ) structure transition through the Burgers path [12]. The four-fold symmetry breaks during the $\mathrm{BCC} \rightarrow \mathrm{HCP}$ transition while the six-fold symmetry breaks during the $\mathrm{HCP} \rightarrow \mathrm{BCC}$ transition, leading to 12 and 3 crystallographic equivalent PTPs and structural states, respectively. Since a crystalline state having both four-fold and six-fold symmetry is theoretically impossible, a high-symmetry state that has all the symmetry elements of these two structures does not exist and the pathway connectivity among the multiple BCC and HCP states cannot be localized 
and limited within the vicinity of any of these states (infinite and interconnected pathway network as will be shown later). The same is true for the face-centered-cubic (FCC) to BCC structural phase transition through the Bain path [13], another commonly observed structural change. Thus Landau theory is limited in a local description and cannot capture the global connectivity of structural states and PTPs as well as the topology of PTG during multiple transition cycles, which is critical for the operation of ferroics. Even though some specific forms of the free energy have been proposed to deal with certain phase transitions (e.g., BCC to $\mathrm{HCP}$ ) in the literature [14], a general way to capture the global connectivity is unavailable. Obviously a new theoretical framework is required to construct PTGs and study their topological features.

Below we formulate a general theoretical framework based on group and graph theories to construct PTGs. Through the construction of PTGs, a fundamental connection among crystal symmetry, topology of PTG and behavior of structural phase transition cycling is established. As examples of applications, typical structural phase transitions found in experiments that offer giant piezoelectricity and giant electro- and magneto-strictions are analyzed and a unique topological feature shared by their PTGs is identified, which reveals the crystallographic requirements of the exceptional functionalities discovered around the so-called morphotropic phase boundaries (MPBs) in these systems [15-23]. Furthermore, we investigated the physical origin of functional fatigue characterized bythe irrecoverable strain accumulated during repeated actuation of SMAs $[24,25]$. We show that the functional fatigue is attributed to the change in PTG topology by the activation of symmetry dictated non-PTPs (NPTP), which leads to the construction of generalized-PTG (GPTG). In particular, crystalline 
defects generated in NiTi because of such a PTG topology change are analyzed and the special grain boundaries and dislocations predicted through the PTG analysis are shown to be consistent with experimental observations. Using PTG topology as a new design criterion, ferroic systems potentially having giant piezoelectricity and giant electro- and magneto-strictions are predicted, and strategies to improve functional fatigue resistance are proposed. Thus PTGs can be used in combination with phase diagrams to motivate and guide the design of ferroic smart materials and other advanced material systems whose properties are controlled by structural phase transition cycling.

\section{Construction of Phase Transition Graph}

Mathematically, a phase transition between two structural states can be interpreted as a pairwise relation and represented conveniently by a graph. Taking advantage of the well-established study of topology (e.g., circular vs. tree, see Fig. 1) and symmetry of a graph in graph theory [26], the topology and symmetry of PTG can be investigated systematically. In particular, the symmetry of a PTG can be defined as the automorphism group of the graph, including all operations that map the PTG onto itself while preserving its connectivity. Note the similarity and difference between crystal symmetry and PTG symmetry; the crystal symmetry is described through space and point groups consisting of all symmetry operations that map a crystal structure onto itself, while the PTG symmetry is described by the automorphism group consisting of all symmetry operations that map the graph onto itself. In fact, a crystal lattice can also be considered as a "lattice graph" with the lattice sites as vertices and the "bonds" between nearest neighboring sites as edges. Then all the operations 
in the space group of the crystal lattice belong to the automorphism group of the "lattice graph". In graph theory, both the topology and symmetry of a graph are important features characterizing its connectivity. They are utilized fully in the current study to analyze and classify PTGs and the associated physical properties of the corresponding phase transition cycling, as will be shown in Section 3.

For a PTG, $G(V, E), V=\left\{v_{\alpha 1}, v_{\alpha 2}, \ldots, v_{\beta 1}, v_{\beta 2}, \ldots\right\}$ is a set of vertices that correspond to the multiple structural states of $\alpha, \beta, \ldots$ phases mentioned earlier and $E$ is a set of edges that connect the vertices and represent the PTPs among the structural states. Since an edge describes a transition process, edges connecting a vertex to itself are excluded. Consider, for example, the PTG for an $\alpha \leftrightarrow \beta$ transition where there are only one structural state for each phase and there is only one PTP between the two structural state. Then the PTG includes two vertices and one edge connecting them. In this case, the order of both $\alpha$ vertex and $\beta$ vertex (i.e., the number of edges connecting to them) is 1 , which can be noted as $\left(N_{\alpha}, N_{\beta}\right)=(1,1)$. In general, $\left(N_{\alpha}, N_{\beta}\right)$ depends on the number PTPs of the forward $\left(N_{\alpha}\right) \alpha \rightarrow \beta$ transition and backward $\left(N_{\beta}\right) \beta \rightarrow \alpha$ transition and can be determined by using group theory $[4,27]$. When $\left(N_{\alpha}, N_{\beta}\right)$ equals $(1, n)$ or $(n, 1)$, a " $n$-star" graph will be generated ( $n$ is an integer larger than 1$)$. However, if both $N_{\alpha}$ and $N_{\beta}$ are larger than 1 for a given $\left(N_{\alpha}, N_{\beta}\right)$, diverse types of PTGs could be generated, including finite or infinite tree graph, and circular graphs with different length, as will be demonstrated below. The global connectivity and topological features of these PTGs depend on the nature of the structural phase transitions. In Figure 1, different types of graphs are classified based to their topology, some of which will be utilized in the following pathway analysis. 
To illustrate the PTG at an intuitive level, we first consider several typical martensitic phase transitions in 2D (Figure 2). For the square $\leftrightarrow$ hexagon transition with the lattice correspondence (LC) shown in Figure 2(a), the vertex order can be determined as $(2,3)[4,27]$. By choosing a reference state (e.g., the square state in the center of Figure 3(a), or $v_{\mathrm{S} 1}$ in Figure 3(b)), all the other structural states or vertices represent different deformation (transformed) states with respect to the reference state (Figure 3(a)) and their connectivity can be determined through a linear algebraic procedure described in Appendix A, leading to the construction of a PTG (Figure 3(b)). Note that the mathematical notation of $v_{\mathrm{S} 1}$ indicates a specific vertex in the graph, which corresponds to a physical structural state of S1 (noted as $\mid \sigma_{S 1}>$ in Appendix A). Note that this PTG is infinite and interconnected. For comparison, the PTGs for the rectangle $\leftrightarrow$ centered-rectangle (diamond) and square $\leftrightarrow$ rectangle (Figures 1(b) and (c)) transitions are also constructed (Figures 2(c) and (d)).

In general, all PTGs involving two phases are $\left(N_{\alpha}, N_{\beta}\right)$-biregular graphs (a specific type of bipartite graphs), which is dictated by the crystal symmetry (both point symmetry and translational symmetry). In graph theory [26], a bipartite graph includes two parts of vertices (i.e., all the vertices can be divided into two disjoint sets) and every edge connects two vertices of different parts. In particular, when all vertices in the same part have the same degree, i.e., the same number of edges connected to them, it constructs a biregular graph (e.g., the bond structure of $\mathrm{SiO}_{2}$ constructs a (4,2)-biregular graph). If we consider all PTPs among all structural states of two phases, then a bipartite PTG emerges and its biregular property is dictated by the crystal symmetry of each phase as well as the symmetry breaking along the 
PTPs. As shown in Figure 3(c), for example, the structural states of $v_{R 1}$ and $v_{R 2}$ are related by a mirror symmetry operation of the crystal lattice, while $v_{R 1}$ and $v_{R 3}$ are related by a lattice invariant shear, which originates from the lattice translational symmetry. In other words, there is a local degeneracy between $v_{R 1}$ and $v_{R 2}$ and a global degeneracy between $v_{R 1}$ and $v_{R 3}$. When two vertices related by the translational symmetry, e.g., $v_{R 1}$ and $v_{R 3}$ in Figure 3(c) and (d), are not connected through a PTP, the PTGs consist of isolated subgraphs. In contrast, when two vertices related by the translational symmetry are connected through a PTP, e.g., $v_{S 1}$ and $v_{S 2}$ in Figure 3(b), the PTG becomes an infinite and interconnected graph. Actually, the crystal structures represented by $v_{S 1}$ and $v_{S 2}$ in Figure $3(\mathrm{~b})$ are both locally and globally equivalent structural states since they are related by both point symmetry (three-fold rotational symmetry when $v_{H 1}$ is taken as a reference) and translational symmetry, which can only be revealed in the framework of PTG. Furthermore, the topological distinction between the isolated subgraphs in Figure 3(c) and (d) is clearly illustrated. The subgraph in Figure 3(d) is a star graph, while that in Figure 3(c) is a circular graph. In graph theory, a star is a tree graph (i.e., a non-circular graph) with one unique vertex as root and all the other vertices as leaves. The degree of each leaf is one. From the graph symmetry point of view, there is no other equivalent state of $v_{S 1}$ (Figure 3(d)) in the isolated subgraph. In other words, $v_{S 1}$ is always a fixed point in any element of the automorphism group of the subgraph. In contrast, there is no such a common fixed point in Figure 3(c). As will be noted below, the PTG including a vertex as a fixed point is actually a critical symmetry feature for a particular type of transition.

Based on the topology of the PTGs, three distinctive types of structural phase transitions can be defined, i.e., 
- Type I: The PTG is an interconnected infinite graph (Figure 3(b));

- Type II: The PTG consists of isolated finite circular subgraphs (Figure 3(c));

- $\quad$ Type III: The PTG consists of isolated finite star subgraphs (Figure 3(d)).

It can be proved easily that the above classification is complete using the graph theory with the equivalence constraints, i.e., the equivalence of all the edges (edge-transitive graph) and the equivalence of all isolated subgraphs (isomorphism of isolated subgraphs), both of which are dictated by the crystal symmetry. The definitions of the three types of transitions are exclusive since their PTG topologies are distinctively different. Typical transitions of Type I include the FCC to BCC transition in iron and its alloys and BCC to HCP transition in titanium and zirconium and their alloys [12]. Because lattice invariant deformations can be realized through PTPs in Type I transitions [13] as shown in Figure 3(b) (e.g., the PTP between $v_{\mathrm{S} 1}$ and $v_{\mathrm{S} 2}$ ), crystalline defects (full dislocations in the square lattice shown in Figure 3(a)) are generated and the infinity of the graph indicates that the defect generation is recurrent during transition cycles (Appendix C). With the special topological feature of an isolated circular graph, Type II transitions can be found, as will be shown below, in ferroelectric and ferromagnetic systems near MPBs, which provide the giant piezoelectric and giant electro-/magneto-strictive properties [15-23, 28-33]. Isolated star subgraphs will be generated for Type III transitions, which are a special type of trees. Because of the existence of the unique vertex as a fixed point, it is the root of a tree, and a transition cycle from a root to a leaf and then back to the root can guarantee a return to the unique root state, leading to transition reversibility and thus offers the shape memory effect. A typical case is the B2 to B19/B19' transition in NiTi-based alloys that are the most widely used SMAs [34]. 
The previous PTG examples can be extended straightforwardly. For example, bipartite graphs between two phases can be generalized easily to $n$-partite graphs among $n$ phases, by which multi-step PTPs like B2-R-B19' in NiTi SMAs could be represented (also see the example in Section 3.2). Although the PTG is initially introduced to describe PTPs, non-phase transition pathways (NPTP) could also be included within the graph framework. In particular, as will be demonstrated later, the topology of a pathway graph may change when easily activated NPTPs are taken into account, so will the fundamental behavior of phase transitions. To avoid any ambiguity, PTGs will refer to pathway graphs containing only PTPs, and the ones consisting of both PTP and NPTP will be referred to as generalized phase transition graph (GPTG). The definition of transition types is based on the topology of PTGs / GPTGs. In Section 3.1, we will keep our discussions within PTGs and show how the giant piezoelectricity and electro-/magneto-striction are dictated by PTG topology. In Section 3.2, we will extend our discussions to GPTG topology. For example, the NiTi system exhibits a good shape memory effect since its PTG (B2 ↔ B19' transition) has an isolated star topology. However, with the NPTPs being included, the GPTG of B2 $\leftrightarrow$ B19' transition becomes infinite and interconnected, so that the system will behave like a Type I transition and generate crystalline defects especially under external loads, leading to functional fatigue. Such a phenomenon can be referred to as "downgrading" of structural transition from Type III to Type I. Another example is a Type III transition that is "downgraded" to Type II transition with the involvement of NPTPs and thus offers giant magnetostriction. In general, giant piezoelectricity and giant magnetostriction can be realized through the NPTPs, i.e., without a phase transition. Since structural state changes along NPTPs are also symmetry breaking 
processes, such as polarization rotation through the NPTPs, they could be well captured and analyzed through the topology their GPTGs (e.g., infinite interconnected, isolated circular or isolated tree graphs). Thus GPTG analysis provides a universal way to describe the symmetry breaking process, no matter it is related to a phase transition or not.

Furthermore, in combination with ab initio calculations [35, 36] of the energy landscapes along different pathways (both PTP and NPTP), weighted and directed graphs could be constructed by labeling weight and direction of each edge, which represents the energy barriers for forward and backward transitions along the pathway. In this way, the physical problem of finding the minimum energy path is reduced to searching for the minimum weight path in a graph, which has been well-addressed in mathematics and computer science [37]. Furthermore, because accessible structural states are identified in PTGs, microstructural domain patterns and defect structures can also be predicted if geometrical constraint of compatibility is incorporated, which parallels exactly those in martensitic transformation crystallography [38-42].

\section{Examples of application of PTG analysis}

In this section, we presented a few typical examples of analyzing the functional properties of ferroic smart materials using their PTG / GPTG topology. In the first example, we consider only PTPs, so that the associated material properties originate solely from phase transitions. In the rest, we generalized our graph theory framework to include both PTPs and NPTPs, so that material properties associated with symmetry breaking processes with or without a phase 
transition can be analyzed, which leads to the one-way "downgrading" rule. Theoretically, both PTG and GPTG analysis are applicable to a given ferroic material. The PTG analysis captures PTPs associated with the symmetry breaking and it requires limited crystallographic information, e.g., crystal structures of the phases involved and lattice correspondence between them. The GPTG analysis, on the other hand, captures all the pathways (both PTPs and NPTPs) associated with symmetry breaking, which usually requires additional information about the possible NPTP pathways (e.g., the identification of a BCO state in NiTi through $a b$ initio calculations) [43]. As will be shown in Section 3.2, the GPTG analysis is necessary especially when material properties are dictated by symmetry breaking processes not directly related to a phase transition, e.g., functional fatigue in $\mathrm{NiTi}$ and giant magnetostriction in $\mathrm{CoFe}[21,44]$.

3.1 Giant piezoelectric and giant electro- / magneto-strictive properties around morphotropic phase boundary in ferroelectric and ferromagnetic materials: PTG analysis

As has been discovered recently in experiments, solid solutions with compositions around the so-called morphotropic phase boundary (MPB) have been identified as a special region that provides giant piezoelectricity of ferroelectric systems such as $\mathrm{PbZrO}_{3}-\mathrm{xPbTO}_{3}(\mathrm{PZT})$ and $\mathrm{PbMg}_{1 / 3} \mathrm{Nb}_{2 / 3} \mathrm{O}_{3}-\mathrm{PbTiO}_{3}$ (PMN-PT) [7, 15-23, 28-33]. Because of the lack of a rigorous theoretical description, however, the crystallographic origin of such giant piezoelectricity is still unclear, and its discovery in existing systems and the continued search for new systems are made more or less by trial-and-error experiments. An MPB is a compositional phase boundary between the tetragonal (T: space group P4mm) and rhombohedral (R: space group 
$R 3 m$ ) phases in metastable phase diagrams of ferroelectric solid solutions. Such metastable phase diagrams capture the relative stabilities of the $\mathrm{C}, \mathrm{T}$ and $\mathrm{R}$ phases with respect to congruent and diffusionless transformations from one phase to another [7, 15, 19]. At or near an MPB, the system is probably a two phase mixture of $\mathrm{T}$ and $\mathrm{R}$ and we hypothesize that the $\mathrm{T} \leftrightarrow \mathrm{R}$ phase transition cycles play a critical role in determining the giant piezoelectricity of MPB systems. Below we construct the PTG for multiple $\mathrm{T} \leftrightarrow \mathrm{R}$ transition cycles, analyze its topological features and identify the ones that could be responsible for the giant piezoelectricity and electro-striction in MPB systems.

As a typical ferroelectric system with MPB, the PZT system involves three structural phase transitions, including cubic (C) (perovskite, paraelectric) $\leftrightarrow \mathrm{T}, \mathrm{C} \leftrightarrow \mathrm{R}$ and $\mathrm{T} \leftrightarrow \mathrm{R}$ transitions (both $\mathrm{T}$ and $\mathrm{R}$ are ferroelectric) [7], but giant piezoelectricity is found only in connection with the $\mathrm{T} \leftrightarrow \mathrm{R}$ transition. The PTGs for the above three phase transitions in PZT are constructed, which are shown in Figure 4. It can be readily seen that both the $\mathrm{C} \leftrightarrow \mathrm{T}$ and $\mathrm{C} \leftrightarrow \mathrm{R}$ transitions belong to Type III while the $\mathrm{T} \leftrightarrow \mathrm{R}$ transition belongs to Type II. Because all the isolated subgraphs in the PTG of the $\mathrm{C} \leftrightarrow \mathrm{T}$ (also for $\mathrm{C} \leftrightarrow \mathrm{R}$ and $\mathrm{T} \leftrightarrow \mathrm{R}$ ) transition are identical, only one subgraph is shown for each transition. In order to show clearly how the PTG is related to crystal symmetry, all the graphs are plotted in 3D. For the $\mathrm{C} \leftrightarrow \mathrm{T}$ transition with vertex order $(6,1)$, there is a tree of one $\mathrm{C}$ vertex as root and six $\mathrm{T}$ vertices as leaves within a subgraph, and the polarization direction of each $\mathrm{T}$ vertex belongs to the $<100>$ type (Figure 4(a)). Similarly, there is one $\mathrm{C}$ root and eight $\mathrm{R}$ leaves with polarization directions of the $<111>$ type in the PTG for the C $\leftrightarrow$ R transition (Figure 4(b)) with vertex order $(8,1)$. In both of the above two cases, no circuits can be found in the PTGs. 
On the contrary, all the six $\mathrm{T}$ vertices and eight $\mathrm{R}$ vertices (with vertex order $(4,3)$ ) are interconnected by multiple circuits in the isolated subgraph in the PTG for the $T \leftrightarrow R$ transition (Figure 4(c)) that takes place at an MPB. The unique topological feature of such a PTG is essential for the giant piezoelectric and giant electro- / magneto-strictive properties discovered at or near an MPB. As demonstrated in the literature, giant piezoelectric and electro- / magneto-strictive responses are driven by easy polarization rotation, which is facilitated by the PTPs of the $\mathrm{T} \leftrightarrow \mathrm{R}$ transition (Figure 4(c)). Suggested by first principle calculations, because of a flat energy surface along the PTPs between $\mathrm{T}$ and R states (i.e., two polar states), a small field will cause a large change of polarization angle $[15,17]$. Such a mechanism is similar to the change from discontinuous phase transition (i.e., first-order phase transition) to continuous (i.e., second-or higher-order) by external fields [11]. As a result, the domain reorientation is realized through continuous polarization rotation rather than nucleation of new domains and subsequent domain boundary migration. In short, the giant piezoelectric and electro- / magneto-strictive properties discovered at or near the MPB originate from continuous polarization rotation through PTPs, the realization of which relies on both a low energy barrier along and the connectivity of the PTPs. Since the former is beyond the scope of this work, we will focus on the connectivity of the PTPs, which is $a$ necessary condition for the polarization rotation mechanism to operate and determined solely by symmetry.

The connectivity of PTPs can be easily captured through the topology of PTG. Considering the topological features of the three types of structural phase transitions, it is obvious that 
Type I transitions should be excluded for ferroic smart material applications because, as mentioned earlier, they are irreversible and inevitably generate defects during the transition cycles due to lattice invariant deformations realized through PTPs (reflected by the infinite, interconnected topology of their PTGs) [13]. To illustrate the effects of the star and circular topologies of PTGs on the transition behavior of a system, we start with several simple examples in 2D. The rectangle to diamond transition (crystal structures shown in Figure 2(b)) is a typical Type II transition and a continuous polarization rotation between any two states (including all rectangle and centered rectangle states) can be guaranteed by its PTG, in which all the vertices are connected through PTPs (Figure 5(a)). When a small external field is applied to a multi-domain system, all the domains (at different states initially) can be reoriented through continuous polarization rotation along the PTPs. In contrast, for a rectangle to parallelogram transition (Type III, Figure 5(c)), continuous polarization rotation through PTPs can only take place within an isolated star (including one rectangle and two parallelogram vertices connected). However, there is no PTP connecting two states in different stars (e.g., between two rectangle states). A state in one star cannot transform to a state in another star through continuous polarization rotation. Another typical Type III transition in 2D is the square to rectangle transition (Figure 5(b)), where polarization rotation cannot be realized through the PTPs because there is no spontaneous polarization in the square state. In fact, all Type III transitions behave like either of the above two cases, which suggests that Type III transitions with PTG topology of isolated star should also be excluded for potential giant piezoelectric and giant electro- / magneto-strictive properties. As a result, giant piezoelectric and giant electro-/magneto-strictive responses through phase transitions can only exist in Type II transitions, which have isolated circular PTGs. As a matter of fact, it has 
been shown experimentally that the piezoelectricity near the T/R boundary is usually an order of magnitude larger than that near the $\mathrm{C} / \mathrm{T}$ or $\mathrm{C} / \mathrm{R}$ boundary in PZT $[15,19]$.

Even though the only phase boundary discovered in literature that has giant piezoelectricity or electric- / magneto-restrictions is between T and R, other boundaries at which PTGs consist of isolated circular subgraphs (i.e., Type II transitions) could also have such properties according to our analysis. For ferroelectric materials, spontaneous polarization can occur only in crystals having a unique polar axis, which belongs to the following 10 polar crystallographic point groups: $1,2, m, m m 2,4,4 m m, 3,3 m, 6,6 m m$ [10]. According to our PTG analysis, giant piezoelectricity, which is associated solely with Type II transitions, cannot be expected when the point group of one phase is 1 because in this case its PTG will be a (n,1)-biregular graph, which indicates a tree subgraph. Also, two phases with 4 (or $4 \mathrm{~mm}$ ) and 6 (or $6 \mathrm{~mm}$ ) point symmetry elements are excluded since an infinite interconnected PTG will be expected. In addition to the R/T phase boundary, another potential MPB for giant piezoelectricity predicted by our analysis is the phase boundary between $\mathrm{R}$ (point group 3 or $3 \mathrm{~m}$ ) and Orthorhombic (point group $\mathrm{mm} 2$ ) phases because it has a PTG with isolated circular subgraphs. As a matter of fact, recent experiments have shown promising giant piezoelectricity for such a system [32].

Applying such PTG analyses to all possible ferroelectric phase transitions (summarized in Figure 6), we identify all the potential systems, in which the piezoelectricity could be greatly enhanced through phase transitions. In addition to the experimentally confirmed $\mathrm{R} / \mathrm{T}$ transition and the newly identified R/O transition discussed above, other transitions that either 
could possibly have (the question marks) or are impossible to have (the cross marks) giant piezoelectricity are also identified in Figure 6. Further determination of the PTG topological features of those ferroelectric transitions with the question marks requires the knowledge of LC (Appendix B). For example, considering a transition between $\mathrm{T}$ and Orthorhombic $(\mathrm{O})$ phases, there could be two possible LCs:

(a) $[100]_{T} \rightarrow[100]_{O},[010]_{T} \rightarrow[010]_{O},[001]_{T} \rightarrow[001]_{O}$ and

(b) $[100]_{T} \rightarrow[100]_{O},[011]_{T} \rightarrow[010]_{O},[0 \overline{1} 1]_{T} \rightarrow[001]_{O}$,

where [001]T is the $c$ axis in the T phase. These LCs will lead to different types of PTGs, with the $\mathrm{T} \leftrightarrow \mathrm{O}$ transition following LC (a) belonging to Type III and that following LC (b) belonging to Type II. As reported in recent experiments [45], the $\mathrm{T}$ to $\mathrm{O}$ transition in (1-x) $\mathrm{BaZr}_{0.2} \mathrm{Ti}_{0.8} \mathrm{O}_{3}-\mathrm{xBa}_{0.7} \mathrm{Ca}_{0.3} \mathrm{TiO}_{3}$ with $\mathrm{LC}$ (b) does offer a giant piezoelectricity, which is consistent with our PTG analysis.

Note that monoclinic (M) phases could play a role in some systems near MPB, as reported in the literature. According to symmetry, $\mathrm{M}$ vertices can be inserted into each edge between $\mathrm{T}$ and R, which yields a new PTG with three phases of R/T/M. However, when the transition between $\mathrm{T}$ and $\mathrm{R}$ (either direct or indirect) exists, isolated circular topology is determined, regardless whether the $\mathrm{M}$ phase exists or not. In this case, continuous polarization rotation can go through a $\mathrm{T} \rightarrow \mathrm{M} \rightarrow \mathrm{R} \rightarrow \mathrm{M} \rightarrow \mathrm{T} \rightarrow \ldots$ path. As a result, the piezoelectric properties could be potentially enhanced through such phase transitions. The predictions in Figure 6 are based on the topology of PTGs containing only PTPs. Similar pathway analysis can also be made with the consideration of NPTP under the same graph theory framework, in which the same mechanism could be realized through NPTP even without a phase transition, as will be shown 
in Section 3.2.

The existence of Type II transitions is also critical for potential giant magnetostriction. From the crystal symmetry identified by using the high accuracy synchrotron x-ray diffractometry, giant magneto-restrictions found in $\mathrm{TDC}\left(\mathrm{TbCo}_{2}-\mathrm{DyCo}_{2}\right)$ system can be related directly to a $\mathrm{T}$ $\leftrightarrow \mathrm{R}$ transition [29], which belongs to Type II based on our classification. Thus the PTG is the same as what is shown in Figure 4(c). Even though the physical origins of electric and magnetic polarizations are fundamentally different, they can be analyzed equally from the viewpoints of crystal symmetry and PTG topology under this new theoretical framework. Note that all our predictions are based solely on crystal symmetry and LC, and the symmetry criterion is universal for the investigation for all kinds of susceptibilities realized through structural phase transitions in ferroic smart materials. In addition to locating interesting phase regions on a phase diagram, critical new information about phase transitions is provided by the PTG analysis, which identifies the types of structural phase transitions and intrinsic properties associated with them.

3.2 Functional fatigue in shape memory alloys and giant responses without phase transition: GPTG analysis

Another important class of ferroic smart materials are SMAs, which are featured by shape memory effect and superelasticity [46-48]. One of the critical issues limiting the service life of SMAs is the so-called functional fatigue during repeated actuation through phase transition cycles. It is found that macroscopic irrecoverable strain can be accumulated in most 
commercial SMAs, leading to the loss of dimensional stability [24, 25, 49]. A large amount of extended crystalline defects such as dislocations and grain boundaries have been observed in typical SMAs including NiTi during the austenite $\leftrightarrow$ martensite transition cycling, induced either by load cycling or thermal cycling (with or without biased load), and the types of defects are identified to be crystallographically specific [24, 44], which suggests a fundamental relationship between functional fatigue and phase transition cycling.

From the symmetry point of view, however, if a group-subgroup relationship exists between the parent and product phases, the martensitic transition (MT) should be fully reversible [13], and if the transformation strain could be well accommodated during the MT and thus local stresses generated by the MT would not cause plastic yielding, then no other lattice defects such as dislocations should be generated. This is obviously not the case even for the widely used commercial SMA, the NiTi system, where defect generation is the rule rather than exception during phase transition cycling [25, 44, 48, 49], especially during thermal cycling under a biased-load much lower than the yield stress. Below we construct PTG and draw connections between the PTG and possible GPTG for the B2 $\leftrightarrow$ B19' transition in NiTi to analyze the evolution of the structural states during multiple transition cycles. In particular, we analyze the topology of the PTG and GPTG as well as the corresponding transition features based on our new classification. Then we predict systematically the characteristic defects generated during the transition cycles and compare them with recent experimental observations.

Following the mathematical procedure given in appendix D, the PTG for the B2 $\leftrightarrow$ B19' 
transition in NiTi is shown in Figure 7, where each B2 state is connected (by the solid lines) with twelve B19' states and each B19' state is connected (by the same solid lines) to only one B2 state, leading to isolated star subgraphs (Type III). However, according to the experimental observations discussed above, the MT in NiTi behaves like Type I, i.e., with plenty of defects generated during transition cycling. To understand what render the Type III MT in NiTi SMA behave like a Type I MT, we search for possible NPTPs that could link these isolated star subgraphs together, forming an interconnected infinite graph. Based on ab initio calculations [43], the B19' structure is both geometrically and energetically close to a base-centered orthorhombic (BCO) structure that has relatively high symmetry comparing to its nearby monoclinic B19' structures. More importantly, according to the symmetry relationship among the BCO, B19' and B2 phases, each BCO state is connected with two B19' states and each B19' state is connected with one B2 state and one BCO state. Thus determined by the symmetry breaking from $\mathrm{BCO}$ to $\mathrm{B} 19$ ', a $\mathrm{BCO}$ state is connected to two $\mathrm{B} 19$ ' states in two different isolated subgraphs and serves naturally as a "bridge" between the two isolated stars. As a result, these symmetry-dictated NPTPs (dash lines in Figure 7) link all the isolated stars into an interconnected and infinite GPTG. Furthermore, it has been shown that the critical stress for the activation of the NPTPs could be as low as $33 \mathrm{MPa}$ [50]. In Figure 7, only four isolated stars are shown to demonstrate their connections by four BCO states (as the bridges) into an interconnected GPTG. Since the GPTG for NiTi is an infinite and interconnected graph when the NPTPs are taken into account, it should behave like a Type I transition and defect generation is anticipated.

Based the PTG, defects generated can be systematically predicted through crystallographic 
analysis. Beside the [100](011) type of dislocations, the generation of special grain boundaries is a unique feature in this system. As calculated in Appendix D, for example, a $\Sigma 9$ boundary is formed between two nearest-neighbor B2 states, and a $\Sigma 5$ boundary is formed between two second-nearest-neighboring B2 states in the GPTG (e.g., the B2 state in the top left and the one in the bottom right of Figure 7), which are generated by the reverse MT from B19'-1 and B19'-10, respectively, back to the two different B2 states via the NPTP (see Figure 7). Based on a recent experimental study of $\mathrm{Ni}_{49.9} \mathrm{Ti}_{50.1}$ under load-biased thermal cycling, various special grain boundaries have been revealed with ASTAR technique after a few transition cycles [44] under a $150 \mathrm{MPa}$ tensile bias load. The generation of special grain boundaries suggests strongly a mechanism distinctively different from the conventional dislocation plasticity and recrystallization $[51,52]$.

From our GPTG analysis, the functional fatigue seems to originate from the existence of NPTPs, which is associated with the translational symmetry of the parent phase crystal lattice. Thus in order to suppress the activation of NPTPs and improve the functional fatigue resistance, the key could be to lower the translational symmetry of the parent phase. Two ways have been proven effective: introducing (a) long range atomic ordering and (b) nano-precipitates in the parent phase [50-53]. As a matter of fact, most commercial reliable SMA systems have ordered parent phases. Similar to atomic ordering, nano-precipitates in the parent phase [53-56] will also break the translational symmetry because of the local lattice distortion and stress concentration caused by the geometrical constraints from the non-transforming precipitates. Besides improving the strength of the parent phase, both of the above design strategies can be considered as the blockage of the NPTPs, which ensures an 
isolated star topology for the PTGs of the systems. It should also be pointed out that dislocation substructures generated during training of SMAs serve a similar purpose.

As demonstrated above, a Type III transition may behave like Type I if its GPTG has infinite and interconnected topology. Similarly, a Type III transition could also behave like Type II if its GPTG has an isolated circular topology. For example, considering a square to rectangle transition (with internal polarization indicated by the arrows in Figure 8) with possible NPTPs (indicated by the dash line in Figure 8) among the rectangular states, an isolated circular GPTG could be constructed, leading to another possibility for obtaining giant susceptibilities by Type III transitions. Similar to the role of the BCO state found in NiTi system, there is a diamond structural state along the NPTP connecting two rectangle states (Figure 8). As a result, the GPTG in Figure 7 is topologically similar to that of the B2 to BCO transition (Type I), while the GPTG in Figure 8 is similar to that of a rectangle to diamond transition (Type II) shown in Figure 5(a). With the consideration of the NPTP in Figure 8, the four rectangle states can form an isolated circuit by themselves. Such a network could occur in low-symmetry triclinic crystals, which may offer giant functionality even without a phase transition. As identified both experimentally and theoretically, such a mechanism through the activation of NPTPs is responsible for the giant responses in several systems [21, 57], which are consistent with our GPTG analysis. As a result, the topology of a GPTG is critical in determining the property of a material system, which is a direct generalization of the classification of Type I/II/III phase transitions based on the PTG topology.

Note that even though NPTPs are defined as pathways connecting structural states of the same 
crystal lattice, e.g., the two B19' states through BCO in Figure 7 and the two rectangular states through the diamond in Figure 8, the symmetry change along the NPTPs in these two examples are fundamentally different. It is associated with the translational symmetry in the former and the point symmetry in the latter. As a result, the inclusion of the NPTP in the former transforms the original isolated star PTG into an interconnected and infinite GPTG while in the latter transforms the original isolated star PTG into an isolated circular GPTG. Thus the topology of a GPTG depends strongly on how the NPTP are connected. The NPTP could be derived by the follow three means: (1) experimental observations of certain transition features (e.g., the generation of characteristic defects such as special grain boundaries in the NiTi system discussed above); (2) geometrical closeness between two structural states of the low symmetry product phase (e.g., the two nearby B19' states in Figure 7); (3) energetic calculations (such as the ab initio calculations [43] discussed above, which have helped us in identifying the NPTPs in NiTi SMA).

From the viewpoint of graph theory, a GPTG has edges more than or equal to those of its corresponding PTG. For a given structural phase transition, a PTG of isolated star topology can be changed to either an isolated circular or an infinite interconnected one so that it is theoretically possible that a Type III transition behaves like Type I/II. A PTG of isolated circular topology can be changed to an infinite interconnected one by adding NPTPs, but cannot be changed to an isolated star, which means that a Type II transition cannot behave like Type I. A PTG of infinite and interconnected topology cannot be changed to an isolated star or circular graph by adding NPTPs. Such a one-way "downgrading” rule could guide the design of ferroic smart materials purely from crystallography even without the knowledge of NPTPs 
activation. For example, one should never expect ferroic functionalities from Type I transition because it is theoretically impossible to be "upgraded" to Type II or Type III. The search for potential systems offering giant piezoelectricity and giant magnetostriction should be around either Type II (e.g., system with MPB) or Type I (e.g., electric/magnetic SMAs). A promising SMA system has to be a Type III transition without easy activation of NPTPs, which might change the topology of PTGs.

\section{Conclusion}

In this paper, a new universal characteristic of structural phase transitions with symmetry-breaking is discovered through the construction of phase transition graphs (PTGs) and the analysis of PTG topology. As a new theoretical concept and framework built upon group and graph theories, the PTG captures the global connectivity of multiple crystallographically equivalent structural states of different phases through phase transition pathways, which dictates the properties and performance of ferroic smart materials in service where multiple forward and backward transition cycles take place. Through analyzing the topology of PTGs, a new classification of structural phase transitions is proposed. An important class of transitions having a unique topological feature, i.e., isolated circular graph that is critical for giant piezoelectricity and giant electro- and magneto-striction in ferroelectric and ferromagnetic materials, are discovered. The physical origin of functional fatigue in shape memory alloys is also analyzed based on the topology of generalized PTG that include symmetry-dictated non-phase transition pathways, and the characteristic defects generated during the phase transition cycling are predicted systematically, which agree well 
with experimental observations in NiTi SMA. This study opens the door for "transition pathway engineering" in ferroic smart materials design by adjusting alloy composition to locate the system at phase boundaries of specific types of structure transitions and by introducing crystalline defects to alter the PTG topology through the activation or deactivation of non-phase-transition pathways for desired properties. In combination with phase diagrams, the PTG analysis will motivate and guide systematic search for high-performance functional and structural materials with much enhanced properties realized through structural phase transition cycling.

\section{Acknowledgement}

We would like to express our sincere wish to Prof. William A. Clark, an expert of crystallography in the Department of Materials Science and Engineering, for a speedy recovery from the violent crime at The Ohio State University. We gratefully acknowledge Prof. Ju Li from MIT for many helpful discussions. The work is support by the National Science Foundation under Grant No. DMR-1410322 and Department of Energy under Grant No. DE-SC0001258. 


\section{Reference}

[1] G. Van Tendeloo, S. Amelinckx, Group-theoretical considerations concerning domain formation in ordered alloys, Acta Cryst. A30 (1974) 431-440.

[2] D. Gratias, R. Portier, M. Fayard and M. Guymont, Crystallographic description of coincidence-site lattice interfaces in homogeneous crystals, Acta Cryst. (1979). A35, 885-894. [3] V. Janovec, T.H. Hahn, H. Klapper, Twinning and domain structures. International table for crystallography (2006), vol. D, Wiley, New York, 2005, pp. 377-392 (Chapter 3.2).

[4] Y. Gao, R. Shi, S. A. Dregia, Y. Wang, Group theory description of transformation pathway degeneracy in structural phase transformations. Acta Mater. 109 (2016), 353-363.

[5] V. K. Wadhawan, Introduction to ferroic materials, Gordon \& Breach, Amsterdam, Netherland, 2000.

[6] J. F. Scott, Applications of modern ferroelectrics. Science 315 (2007), 954-959.

[7] B. Jaffe, W. R. Cook, H. Jaffe, Piezoelectric Ceramics, Academic Press Inc., London, UK, 1971.

[8] X. Ren, Large electric-field-induced strain in ferroelectric crystals by point-defect-mediated reversible domain switching. Nat. Mater. 3 (2004), 91-94.

[9] S. M. Ueland, Y. Chen, C. A. Schuh, Oligocrystalline shape memory alloys. Adv. Funct. Mater. 22 (2012), 2094-2099.

[10] J. F. Nye, Physical Properties of Crystals: Their Representation by Tensors and Matrices, Oxford University Press, USA, 1985.

[11] L.D. Landau, On the theory of phase transitions. Phys. Z. Sowjetunion 11 (1937), 545.

[12] W. G. Burgers, On the process of transition of the cubic-body-centered modification into the hexagonal-close-packed modification of zirconium. Physica 1 (1934), 561-586. 
[13] K. Bhattacharya, S. Conti, G. Zanzotto, J. Zimmer, Crystal symmetry and the reversibility of martensitic transformations. Nature 428 (2004), 55-59.

[14] P. Toledano, V. Dmitriev, Reconstructive phase transitions in crystals and quasicrystals. World Scientific Pub Co Inc, 1996.

[15] M. Ahart, M. Somayazulu, R.E. Cohen, P. Ganesh, P. Dera, H. Man, R. J. Hemley, Y. Ren, P. Liermann, Z. Wu, Origin of morphotropic phase boundaries in ferroelectrics. Nature 451 (2008), 545-548.

[16] B. Xu, D. Wang, J. Iniguez, L. Bellaiche, Finite - temperature properties of rare - earth substituted $\mathrm{BiFeO}_{3}$ multiferroic solid solutions. Adv. Funct. Mater. 25 (2015), 552-558.

[17] H. Fu, R. E. Cohen, Polarization rotation mechanism for ultrahigh electromechanical response in single-crystal piezoelectrics. Nature 403 (2000), 281-283.

[18] F. Ma, P. Liu, W. Li, X. Liu, X. Chen, K. Zhang, D. Pan, W. Lu, The mechanical behavior dependence on the $\mathrm{TiB}$ whisker realignment during hot-working in titanium matrix composites. Sci. Rep. 6 (2016), 36126.

[19] A. G. Khachaturyan, Ferroelectric solid solutions with morphotropic boundary: Rotational instability of polarization, metastable coexistence of phases and nanodomain adaptive states. Phil. Mag. 90 (2010), 37-60.

[20] Z. Kutnjak, J. Petzelt, R. Blinc, The giant electromechanical response in ferroelectric relaxors as a critical phenomenon, Nature 441 (2006), 956-959.

[21] D. Hunter, W. Osborn, K. Wang, N. Kazantseva, J. Hattrick-Simpers, R. Suchoski, R. Takahashi, M. L. Young, A. Mehta, L. A. Bendersky, S. E. Lofland, M. Wuttig, I. Takeuchi, Giant magnetostriction in annealed $\mathrm{Co}_{1-\mathrm{x}} \mathrm{Fe}_{\mathrm{x}}$ thin-films. Nat. Comm. 5 (2011), 518.

[22] M. Porta, T. Lookman, Effects of tricritical points and morphotropic phase boundaries on 
the piezoelectric properties of ferroelectrics. Phys. Rev. B 83 (2011), 174108.

[23] J. Ouyang, J. Slusker, I. Levin, D. -M. Kim, C. -B. Eom, R. Ramesh, A. L. Roytburd, Engineering of self - assembled domain architectures with ultra - high piezoelectric response in epitaxial ferroelectric films. Adv. Funct. Mater. 17 (2007), 2094-2100.

[24] G. Eggeler, E. Hornbogen, A. Yawny, A. Heckmann, M. Wagner. Structural and functional fatigue of NiTi shape memory alloys. Mater. Sci. Eng. A 378 (2004), 24-33.

[25] S. V. Raj \& R. D. Noebe. Low temperature creep of hot-extruded near-stoichiometric NiTi shape memory alloy part II: Effect of thermal cycling. Mater Sci. Eng. A 581 (2013), 154-163.

[26] F. Harary, Graph Theory, Addison-Wesley, Reading, MA, USA 1994.

[27] J. W. Cahn, The Symmetry of Martensites. Acta Metall. 25 (1977), 721-724.

[28] Z. Chen, Z. Luo, C. Huang, Y. Qi, P. Yang, L. You, C. Hu, T. Wu, J. Wang, C. Gao, T. Sritharan, L. Chen, Low - symmetry monoclinic phases and polarization rotation path mediated by epitaxial strain in multiferroic $\mathrm{BiFeO}_{3}$ thin films. Adv. Funct. Mater. 21 (2011), 133-138.

[29] S. Yang, H. Bao, C. Zhou, Y. Wang, X. Ren, Y. Matsushita, Y. Katsuya, M. Tanaka, K. Kobayashi, X. Song, J. Gao, Large magnetostriction from morphotropic phase boundary in ferromagnets. Phys. Rev. Lett. 104 (2010), 197201.

[30] V. M. Goldschmidt, Crystal structure and chemical constitution. A lecture delivered before the Faraday Society on Thursday, 14th March, 1929. Trans. Faraday Soc. 1929, 25, 253.

[31] X. Gao, Z. Wi, F. Zhao, Y. Yang, R. Chen, X. Fang, J. Tang, D. Fang, D. Wang, R. Li, X. Ge, X. Ma, X. Wang, Investigation of localized states in GaAsSb epilayers grown by 
molecular beam epitaxy. Sci. Rep. 6 (2016), 29112.

[32] Y. Yao, C. Zhou, D. Lv, D. Wang, H. Wu, Y. Yang, X. Ren, Large piezoelectricity and dielectric permittivity in $\mathrm{BaTiO}_{3}-\mathrm{xBaSnO}_{3}$ system: The role of phase coexisting. Europhys. Lett. 98 (2012), 2.

[33] F. Ma, T. Wang, P. Liu, W. Li, X. Liu, X. Chen, D. Pan, W. Lu, Mechanical properties and strengthening effects of in situ $(\mathrm{TiB}+\mathrm{TiC}) / \mathrm{Ti}-1100$ composite at elevated temperatures, Mater. Sci. \& Eng. A. 654 (2016) 352-358.

[34] K. Otsuka, X. Ren, Physical metallurgy of Ti-Ni-based shape memory alloys. Prog. Mater. Sci. 50 (2005), 511-678.

[35] R. G. Hennig, D. R. Trinkle, J. Bouchet, S. G. Srinivasan, R. C. Albers, J. W. Wilkins, Impurities block the $\alpha$ to $\omega$ martensitic transformation in titanium. Nat. Mater. 4 (2005), $129-133$.

[36] K. J. Caspersen, E. A. Carter, Finding transition states for crystalline solid--solid phase transformations. Proc. Natl. Acad. Sci. 102 (2005), 6738-6743.

[37] T. H. Cormen, C. E. Leiserson, R. L. Rivest,C. Stein, Introduction to Algorithms, MIT Press, Cambridge, USA, 2001.

[38] M. S. Wechsler, D. S. Lieberman \& T. A. Read. On the theory of the formation of martensite. Trans. AIME 197 (1953), 1503-1515.

[39] J. K. Mackenzie \& J. S. Bowles. The crystallography of martensite transformations II. Acta Metall. 2 (1954), 138-147.

[40] C. M. Wayman. Introduction to the crystallography of martensitic transformation Collier-Macmillan, New York, 1964.

[41] A. G. Khachaturyan, Theory of Structural Transformations in Solids, Wiley, New York, 
USA, 1983.

[42] K. Bhattacharya, Microstructure of Martensite: Why it Forms and How it Gives Rise to the ShapeMemory Effect, Oxford Univ. Press, Oxford, UK, 2003.

[43] X. Huang, G. J. Ackland, K. M. Rabe. Crystal structures and shape-memory behaviour of NiTi. Nat. Mater. 2 (2003), 307-311.

[44] M. L. Bowers, Y. Gao, L. Yang, D. J. Gaydosh, M. De Graef, R. D. Noebe, Y. Wang, M. J. Mills. Austenite grain refinement during load-biased thermal cycling of a Ni49.9Ti50.1 shape memory alloy. Acta Mater. 91 (2015), 318-329.

[45] L. Zhang, M. Zhang, L. Wang, C. Zhou, Z. Zhang, Y. Yao, Li. Zhang, D. Xue, X. Lou, X. Ren, Phase transitions and the piezoelectricity around morphotropic phase boundary in $\mathrm{Ba}$ $\left(\mathrm{Zr}_{0.2} \mathrm{Ti}_{0.8}\right) \mathrm{O}_{3}-\mathrm{x}\left(\mathrm{Ba}_{0.7} \mathrm{Ca}_{0.3}\right) \mathrm{TiO}_{3}$ lead-free solid solution. Appl. Phys. Lett. 105 (2014), 162908. [46] M. D. Cai, S. C. Langford SC, M. J. Wu, W. Huang, G. Xiong, T. C. Droubay, A.G. Joly, K. M. Beck, W. P. Hess, J. T. Dickinson, Study of martensitic phase transformation in a $\mathrm{NiTiCu}$ thin-film shape-memory alloy using photoelectron emission microscopy. Adv. Funct. Mater. 17 (2007), 161-167.

[47] M. Zarinejad, Y. Liu, Dependence of transformation temperatures of NiTi - based shape - memory alloys on the number and concentration of valence electrons. Adv. Funct. Mater. 18 (2008), 2789-2794.

[48] S. Miyazaki, Y. Igo, K. Otsuka. Effect of thermal cycling on the transformation temperatures of Ti-Ni alloys. Acta Metall. Mater. 34 (1986), 2045-2051.

[49] A. R. Pelton, G. H. Huang, P. Moine, R. Sinclair. Effects of thermal cycling on microstructure and properties in Nitinol. Mater. Sci. Eng. A 532 (2012), 130-138.

[50] S. Rajagopalan, A. L. Little, M. A. M. Bourke, R. Vaidyanathan. Elastic modulus of 
shape-memory NiTi from in situ neutron diffraction during macroscopic loading, instrumented indentation, and extensometry. Appl. Phys. Lett. 86 (2005), 081901.

[51] J. P. Hirth, J. Lothe, Theory of Dislocations, Wiley, New York, 1982.

[52] R. D. Doherty, D. A. Hughes, F. J. Humphreys, J.J. Jonas, D.Juul Jenson. M. E.Kassner, W. E. King, T. R. McNelley, H. J. McQueen, A. D. Rollett, Current issues in recrystallization: a review. Mater. Sci. Eng. A 238 (1997), 219-274.

[53] K. Otsuka \& K. Shimizu. On the crystallographic reversibility of martensitic transformations. Scripta Metall. 11 (1977), 757-760.

[54] Y. Gao, N. Zhou, F. Yang, Y. Cui, L. Kovarik, N. Hatcher, R. D. Noebe, M. J. Mills, Y. Wang. P-phase precipitation and its effect on martensitic transformation in $(\mathrm{Ni}, \mathrm{Pt}) \mathrm{Ti}$ shape memory alloys. Acta Mater. 60 (2012), 1514-1527.

[55] J. Khalil-Allafi, A. Dlouhy, G. Eggeler. Ni4Ti3-precipitation during aging of NiTi shape memory alloys and its influence on martensitic phase transformations. Acta Mater 50 (2002), $4255-4274$.

[56] K. Gall, H. Sehitoglu, Y. I. Chumlyakov, I. V. Kireeva, H. J. Maier. The influence of aging on critical transformation stress levels and martensite start temperatures in NiTi: Part II-discussion of experimental results. J. Eng. Mater. Technol. 121 (1999), 128-37.

[57] W. F. Rao, M. Wuttig, A. G. Khachaturyan, Giant nonhysteretic responses of two-phase nanostructured alloys. Phys. Rev. Lett. 106 (2011), 105703. 


\section{Appendix A. Construction of phase transition graph (PTG)}

To make a non-localized description of phase transition network, mathematical expressions of structural states and phase transition pathways (PTPs) are first presented based on phase transformation crystallography [38-42], which are mapped to vertices and edges, respectively, leading to the construction of PTGs.

Without loss of generality, the structural change between two structural states along a PTP is described by an operator $\hat{\mathbf{T}}$, and a compact algebraic description (bra-ket notation used in quantum mechanics) similar to that used in martensitic transformation crystallography $[38,39]$ is adapted here. Each structural state is noted by a high-dimensional vector $\left|\sigma_{i}\right\rangle$, which can be represented in a basis of $C_{i}$ as $\left\langle C_{i} \mid \sigma_{i}\right\rangle$. The transition from structural state $\left|\sigma_{i}\right\rangle$ to $\left|\sigma_{j}\right\rangle$ is described by an operator, $\hat{\mathbf{T}}_{j, i}$, which satisfies,

$$
\left|\sigma_{j}>=\hat{\mathbf{T}}_{j, i}\right| \sigma_{i}>
$$

In general, $\hat{\mathbf{T}}$ could operate on several structural order parameters that define the structural states of parent and product phases, including electric and magnetic polarizations, depending on the intrinsic feature of a transition. Particularly when $\hat{\mathbf{T}}_{j, i}$ describes a uniform deformation in $3 \mathrm{D},\left|\sigma_{i}\right\rangle$ is simply expressed by three linear-independent $3 \mathrm{D}$ lattice vectors, $\mid \sigma_{i}>=\left\{\mathbf{e}_{1}^{i}, \mathbf{e}_{2}^{i}, \mathbf{e}_{3}^{i}\right\}$, which can be represented in any basis. Because $\hat{\mathbf{T}}_{j, i}$ is a second-rank tensor connecting two states, two basis indicators are needed for its representation. Such a notation is especially convenient for sequential changes among different structural states, which is described by exertion of a series of operators. Note that Eqn. (A.1) is dictated by the correspondence between $\left|\sigma_{i}\right\rangle$ and $\left|\sigma_{j}\right\rangle$ (or the lattice correspondence between $\left\{\mathbf{e}_{1}^{i}, \mathbf{e}_{2}^{i}, \mathbf{e}_{3}^{i}\right\}$ and $\left.\left\{\mathbf{e}_{1}^{j}, \mathbf{e}_{2}^{j}, \mathbf{e}_{3}^{j}\right\}\right)$, and $\hat{\mathbf{T}}$ is a characteristic operator of the phase transition. 
The above mathematical description of structural transitions can be mapped onto a PTG, $G(V, E) . V$ is a set of vertices that correspond to different structural states of $\alpha, \beta, \ldots$ phases and $E$ is a set of edges that connect the vertices and describes PTPs among them. Consider a PTG for the $\alpha \leftrightarrow \beta$ transition. The order of each vertex (number of edges connecting to it) depends on PTP degeneracy that can be determined using group theory [4, 27]. For each vertex $i$, its state can be described by $\left|\sigma_{i}\right\rangle=\left\{\mathbf{e}_{1}^{i}, \mathbf{e}_{2}^{i}, \mathbf{e}_{3}^{i}\right\}$ in the way that the lattice correspondence (LC) among all the structural states is guaranteed. After defining a basis $C_{i}$ for each state, all the edges can be written in matrix forms $\left\langle C_{j}\left|\mathbf{T}_{j, i}\right| C_{i}>\right.$ through Eqn. (A.1):

$$
<C_{j}\left|\sigma_{j}>=<C_{j}\right| \mathbf{T}_{j, i}\left|C_{i}><C_{i}\right| \sigma_{i}>
$$

Note that the determination of $<C_{j}\left|\mathbf{T}_{j, i}\right| C_{i}>$ requires $<C_{i} \mid \sigma_{i}>$ and $<C_{j} \mid \sigma_{j}>$. Since each of them is calculated within its own basis (i.e., $\left|\sigma_{i}\right\rangle$ in $C_{i},\left|\sigma_{j}\right\rangle$ in $C_{j}$ ), there is no direct constraint about the spatial relationship between $C_{i}$ and $C_{j}$. Only the correspondence between $\left|\sigma_{i}\right\rangle$ and $\left|\sigma_{j}\right\rangle$ is required. To obtain a unique representation for each vertex, a reference state $\left|\sigma_{0}\right\rangle$ (represented in $C_{0}$ basis) is chosen, which can be considered as the "undeformed state", and all the other states can be expressed with respect to it:

$$
\left|\sigma_{i}>=\hat{\mathbf{T}}_{i, 0}\right| \sigma_{0}>
$$

Note that there is a fundamental difference between $\hat{\mathbf{T}}_{i, 0}$ and $\hat{\mathbf{T}}_{j, i} \cdot \hat{\mathbf{T}}_{i, 0}$ characterizes the deformation state of $\left|\sigma_{i}\right\rangle$ with respect to the reference state $\left|\sigma_{0}\right\rangle$ (not necessarily a vertex), while $\hat{\mathbf{T}}_{j, i}$ characterizes the edge between $\left|\sigma_{i}\right\rangle$ and $\left|\sigma_{j}\right\rangle$ through a PTP. As a result, all the vertices can be expressed in matrix forms $<C_{i}\left|\mathbf{T}_{i, 0}\right| C_{0}>$, and two vertices connected by an edge, or two states connected by a PTP, can be described as: 


$$
<C_{j}\left|\mathbf{T}_{j, 0}\right| C_{0}>=<C_{j}\left|\mathbf{T}_{j, i}\right| C_{i}><C_{i}\left|\mathbf{T}_{i, 0}\right| C_{0}>
$$

Starting from one vertex $\left|\sigma_{i}\right\rangle$ expressed as $\left\langle C_{i}\left|\mathbf{T}_{i, 0}\right| C_{0}>\right.$ in $C_{i}$ basis, a number of pathways are determined as $\hat{\mathbf{T}}_{j, i} \mid C_{i}>$. For another known vertex $\left|\sigma_{k}\right\rangle$, it is connected to $\left|\sigma_{i}\right\rangle$ if and only if it has the exact same matrix representation as $\left\langle C_{j}\left|\mathbf{T}_{i, 0}\right| C_{0}>\right.$ in the same basis of $C_{j}$,

$$
<\left.C_{j}\right|_{k}>=<\left.C_{j}\right|_{j}>=<C_{j}\left|\mathbf{T}_{j, i}\right| C_{i}><C_{i}\left|\mathbf{T}_{i, 0}\right| C_{0}>
$$

which indicates that $\left|\sigma_{k}\right\rangle$ is actually $\left|\sigma_{j}\right\rangle$. According to the above equation, the connectivity between any two vertices can be determined unambiguously, leading to the construction of a PTG.

\section{Appendix B. Effect of lattice correspondence on PTG topology}

As a new approach to investigating crystallography and symmetry breaking during structural phase transitions, the PTG characterizes the symmetry and topology of phase transition network, which represents a deeper intrinsic symmetry character beyond crystal structure. Note that A PTG is determined solely by the crystal structures of the parent and product phases and the LC between them. LC is a critical piece of information required to determine the vertex order and global connectivity. For example, considering the transition between a rectangle and a centered-rectangle, the vertex order of its PTG is $(2,2)$ according to the LC shown in Figure. 1(b), which forms an isolated circuit topology (Figure 3(c)). However, if another LC as shown in Figure S1(a) is assumed, an infinite chain topology is formed, even though the vertex order is also $(2,2)$. As a result, the local pathway degeneracies of the two cases determined by group theory are identical. However, distinctions between these two examples with the same parent and product phases but different LCs can be illustrated clearly by the non-localized PTGs that are beyond the reach of local symmetry breaking captured by 
group theory (i.e., one forms an isolated circuit while the other forms an infinite chain). As indicated in Figure 6, the cross marks suggest that the type of the transition cannot be Type II regardless of LC, while the question marks suggest that the type of the transition depends on LC, which could be Type II.

\section{Appendix C. Defect structures generated during a Type I transition.}

Because lattice invariant deformation can be realized through PTPs in Type I transitions, defect structures can be generated during such transitions, which are originated from translational symmetry.

As shown in Figure 3(a)\&(b), a PTG for a 2D square to hexagonal transition has been constructed. In particular, assuming that the lattice constants for both square and hexagonal lattices are identical, i.e., $a^{S}=a^{H}, v_{S 1}$ is chosen as the reference state $\left(\left|v_{S 1}>=\right| 0>\right)$ and an orthogonal basis is chosen as $C_{S 1}=\left\{\mathbf{e}_{1}^{\alpha}, \mathbf{e}_{2}^{\alpha}\right\}$ (Figure 2(a)), all the operators can be represented as $2 \times 2$ matrices, some of which are listed as the followings:

$$
\begin{aligned}
& <C_{S 1}\left|\mathbf{T}_{S 1,0}\right| C_{S 1}>=\left[\begin{array}{ll}
1 & 0 \\
0 & 1
\end{array}\right] \\
& <C_{S 1}\left|\mathbf{T}_{H 1,0}^{+} \mathbf{T}_{H 1,0}\right| C_{S 1}>=\left[\begin{array}{cc}
1 & 1 / 2 \\
1 / 2 & 1
\end{array}\right] \\
& <C_{S 1}\left|\mathbf{T}_{S 2,0}^{+} \mathbf{T}_{S 2,0}\right| C_{S 1}>=\left[\begin{array}{ll}
1 & 1 \\
1 & 2
\end{array}\right]
\end{aligned}
$$


In the followings, we will focus on the relationship between neighboring vertices. Considering the relationship between two vertices for the square lattice which have a common neighbor of hexagonal vertex (Figure 3(b)), i.e., $v_{S 1}$ and $v_{S 2}$, defect structures can be determined by applying compatibility constraint (or invariant plane strain condition in martensitic transformation crystallography) [38-42],

$$
\mathbf{R T}_{S 2,0}-\mathbf{T}_{S 1,0}=\mathbf{b}^{S} \otimes \mathbf{n}^{S}
$$

where $\mathbf{R}$ is a rigid-body rotation, and $\otimes$ is the operator of dyadic product. $\mathbf{b}^{S}$ and $\mathbf{n}^{S}$ are the shear vector and shear plane normal of corresponding lattice invariant shear deformation.

By solving Eqn. (C.4), two solutions can be obtained as follows,

$$
\begin{aligned}
& \left\{\begin{array}{l}
\mathbf{b}_{1}^{S}=\left(\begin{array}{l}
1 \\
0
\end{array}\right) \\
\mathbf{n}_{1}^{S}=\left(\begin{array}{l}
0 \\
1
\end{array}\right)
\end{array}\right. \\
& \left\{\begin{array}{l}
\mathbf{b}_{2}^{S}=\frac{1}{5}\left(\begin{array}{c}
-1 \\
2
\end{array}\right) \\
\mathbf{n}_{2}^{S}=\left(\begin{array}{l}
2 \\
1
\end{array}\right)
\end{array}\right.
\end{aligned}
$$

From the first solution, it can be concluded that the structural state of $\left|v_{S 2}\right\rangle$ is equivalent to that of a dislocation loop inside $\left|v_{S 1}\right\rangle$ with Burger vector $\mathbf{b}_{1}^{S}$ and slip plane normal $\mathbf{n}_{1}^{S}$, which are represented in the $\mid 0>$ basis (Figure C.1).

And in the second solution, $\mathbf{R}$ is corresponding to a $53.13^{\circ}$ rotation along [001], which leads to a $\Sigma 5$ grain boundary (Figure C.2). Actually, $\left|v_{S 2}\right\rangle$ and $\left|v_{S 1}\right\rangle$ are related by a lattice invariant 
deformation which can be realized through two edges of PTPs. Such a relationship is usually described as twinning with respect to state $\left|v_{H 1}\right\rangle$. And defect structures given by the above two solutions can be interpreted as different types of twinning. However, if they are considered in general point of view, all the crystalline defects including twinning, dislocation and grain boundary are originated from symmetry breaking during phase transitions. Such a mechanism is general in all the structural phase transitions of Type I, and the type of defects can be determined through Eqn. (C.4).

\section{Appendix D. Calculations of special grain boundary generation during transition cycling}

\section{in NiTi shape memory alloys}

The defect structures generated by this Type I GPTG can be predicted by using the phenomenological theory of martensite crystallography (PTMC) [38-42]. Considering two "neighboring" B2 states (two B2-centered stars connected through one BCO, e.g., the two B2 states in the bottom of Figure 6), for example, the deformation gradient matrix from one B2 to the other B2 can be determined as:

$$
\mathbf{T}=\left[\begin{array}{ccc}
1 & 0.5 & 0.5 \\
0 & 1 & 0 \\
0 & 0 & 1
\end{array}\right]
$$

The characteristic defect structures during the transition can be predicted by incorporating the geometrical constraint of compatibility (or invariant plane strain condition) [38-42],

$$
\mathbf{Q T}=\mathbf{I}+\mathbf{a} \otimes \mathbf{n}
$$

where $\mathbf{Q}$ is a rigid body rotation, and $\mathbf{Q T}$ is an invariant plane strain. $\mathbf{n}$ is the normal of the invariant plane and $\mathbf{a}$ is the displacement that characterizes the shape change (uniform lattice distortion associated with the invariant plan strain. Two solutions can be obtained, 


$$
\left\{\begin{array} { l } 
{ \mathbf { a } _ { 1 } = \frac { 1 } { 2 } ( \begin{array} { l } 
{ 1 } \\
{ 0 } \\
{ 0 }
\end{array} ) } \\
{ \mathbf { n } _ { 1 } = ( \begin{array} { l } 
{ 0 } \\
{ 1 } \\
{ 1 }
\end{array} ) }
\end{array} \quad \text { and } \quad \left\{\begin{array}{l}
\mathbf{a}_{2}=\frac{1}{18}\left(\begin{array}{c}
-1 \\
2 \\
2
\end{array}\right) \\
\mathbf{n}_{2}=\left(\begin{array}{l}
4 \\
1 \\
1
\end{array}\right)
\end{array}\right.\right.
$$

The first solution describes the [100](011) type of dislocations in B2 NiTi. The second solution suggests a relatively high index plane (411) and the rotation $\mathbf{Q}$ in Eqn. (D.2) is a $38.94^{\circ}$ rotation around the [0 $\left.\overline{1} 1\right]$ direction, which is the characteristic angle of a $\Sigma 9$ boundary. A simple illustration of the two solutions can be made in parallel with the prediction of plate martensite in classical martensitic crystallography [38-42]. The first solution can be interpreted as a single dislocation loop of [100](011) type in a B2 crystal, while the second solution can be interpreted as an isolated plate-shaped grain (with $38.94^{\circ}$ misorientation) embedded in another large grain. Both of them are parallel exactly to a plate-shaped martensite embedded in an austenite matrix. However, because of the spatial correlation (e.g., elastic interaction) of multi-domain and defect structures, a rather complicated microstructure is observed in experiments [44], including complex dislocation networks as well as various types of grain boundaries.

In a similar way, all the possible defect structures generated during a single phase transition cycle can be determined. For example, considering the deformation between two second-nearest-neighboring B2 states in the GPTG (e.g., the B2 state in the top left and the one in the bottom right of Figure 6), which are generated by the reverse MT from B19'-1 and B19'-10, respectively, back to the two different B2 states via the NPTP, one finds a $\Sigma 5$ boundary. Considering the entire first and second nearest neighbor B2 relationships in the 
GPTG, one finds $\Sigma 3, \Sigma 5, \Sigma 7, \Sigma 9$ and $\Sigma 13$. The type of special grain boundaries generated is dictated by the choice of pathways, which will be affected by both external stress and internal local stress concentration in multi-domain microstructures generated during the martensitic transformation.

Through similar computational crystallography analysis, higher order special grain boundaries such as $\Sigma 11, \Sigma 15, \Sigma 17, \ldots, \Sigma 37, \Sigma 39, \ldots$ can be generated after two cycles (considering the third and fourth nearest-neighboring B2 states), three cycles (considering the fifth and sixth nearest-neighboring B2 states), ..., which can be determined systematically. Based on a recent experimental study of $\mathrm{Ni}_{49.9} \mathrm{Ti}_{50.1}$ under load-biased thermal cycling, various special grain boundaries have been revealed with ASTAR technique after a few transition cycles under a $150 \mathrm{MPa}$ tensile bias load [44]. The generation of special grain boundaries suggests strongly a mechanism associated with crystal symmetry and phase transition and not expected in conventional dislocation plasticity and recrystallization. Based on the above theoretical analysis, the types of the defects are dictated by symmetry-breaking along the PTPs and NPTPs, which can be evaluated systematically using the framework of PTG and GPTG. 


\section{Figure Captions}

Figure 1. Topological classification of infinite graphs.

Figure 2. Example of structural phase transitions leading to different types of PTG topology: (a) square to hexagonal; (b) rectangle to centered-rectangle; (c) square to rectangle. (Lattice correspondences are indicated by the basis vectors of the same subscripts in these drawings)

Figure 3. Construction of Phase Transition Graph (PTG) in 2D: (a) structural states and (b) PTG for the square to hexagonal transition (Blue and red line in (a) represent two independent vectors in a 2D lattice and they also indicate the lattice correspondence between two structure states); (c) PTG for the rectangle to centered-rectangle transition; (d) PTG for the square to rectangle transition.

Figure 4. Topology of isolated subgraph in PTG for phase transitions in PZT ferroelectrics: (a) cubic to tetragonal; (b) cubic to rhombohedra; (c) tetragonal to rhombohedra. (The polarization directions are indicated by arrows)

Figure 5. PTG graphs for ferroelectric transitions among square, rectangle, parallelogram and centered rectangle lattices. The polarization direction is indicated by the black arrows. (a) rectangle to centered rectangle; (b) square to rectangle; (c) rectangle to parallelogram.

Figure 6. Potential phase transitions that could enhance piezoelectric properties. (Further information about lattice correspondence is required to determine the systems with question 
marks)

Figure 7. PTG (with PTPs only) and GPTG (with both PTPs and NPTPs) for the B2 to B19' transition in NiTi shape memory alloys (solid line: PTP, dash line: NPTP).

Figure 8. PTG (with PTPs only) and GPTG (with both PTPs and NPTPs) for a rectangle to centered rectangle transition in 2D. (solid line: PTP, dash line: NPTP). The Diamond structure along the NPTP is indicated.

Figure B.1. Crystal structures and PTGs for a square to rectangle transition (LC is indicated by the correspondence among boxes outlined by the solid lines, while the unit cells are outlined by the dashed lines).

Figure C.1. Dislocation generation during a 2D hexagonal to square transition: (a) Perfect crystal of hexagonal lattice (in structural state HEX-1 indicted by $v_{H 1}$ ); (b) dislocation loop in square lattice (part of material circled by red lines undergoes $v_{H 1} \rightarrow v_{S 1}$, while part of material circled by green lines undergoes $v_{H 1} \rightarrow v_{S 2}$ ).

Figure C.2. Grain generation during a 2D hexagonal to square transition: (a) Perfect crystal of hexagonal lattice (in structural state HEX-1 indicted by $v_{H 1}$ ); (b) dislocation loop in square lattice (part of material circled by red lines undergoes $v_{H 1} \rightarrow v_{S 1}$, while part of material circled by green lines undergoes $v_{H 1} \rightarrow v_{S 2}$ ). 


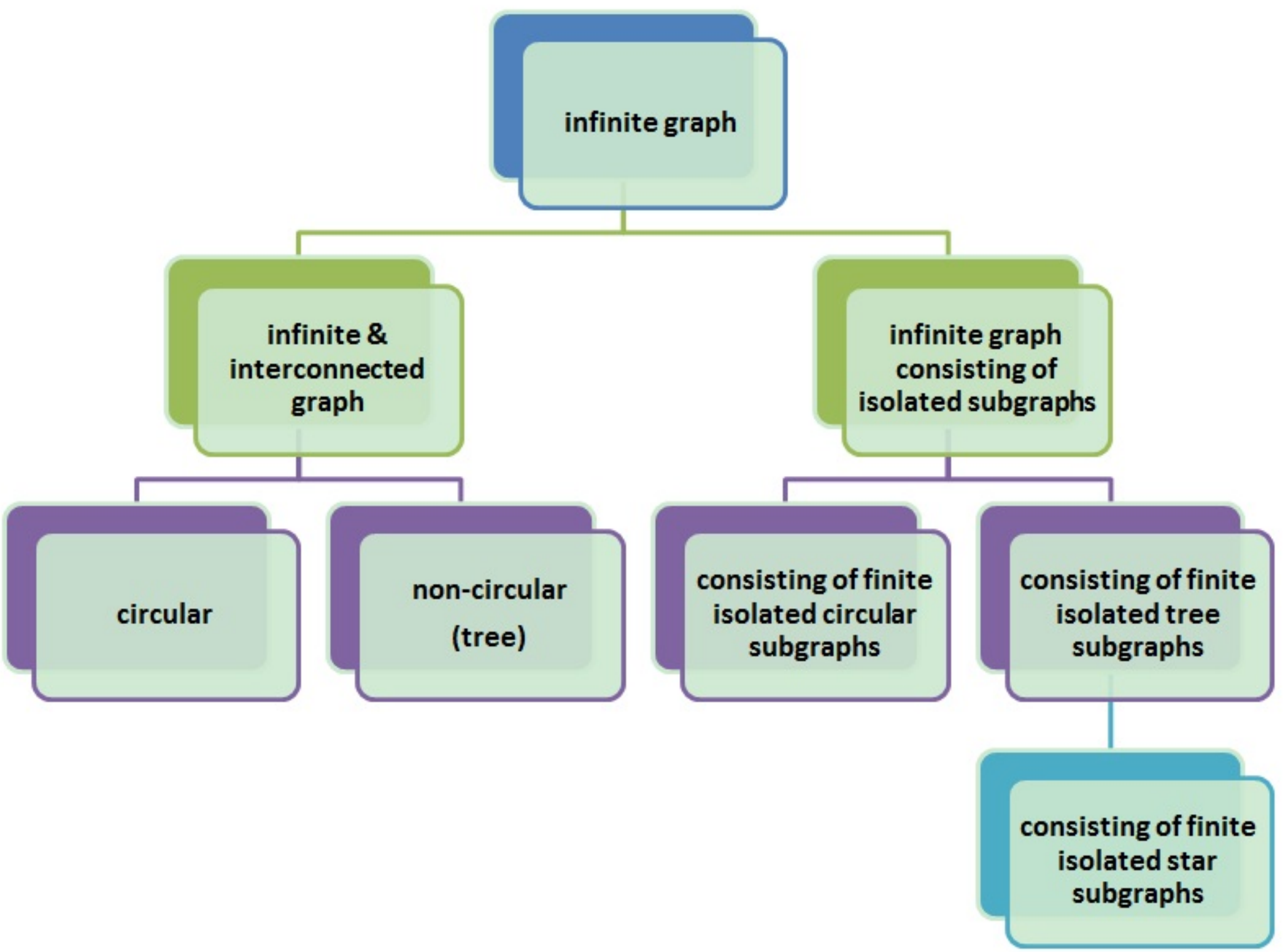


(a)

(b)
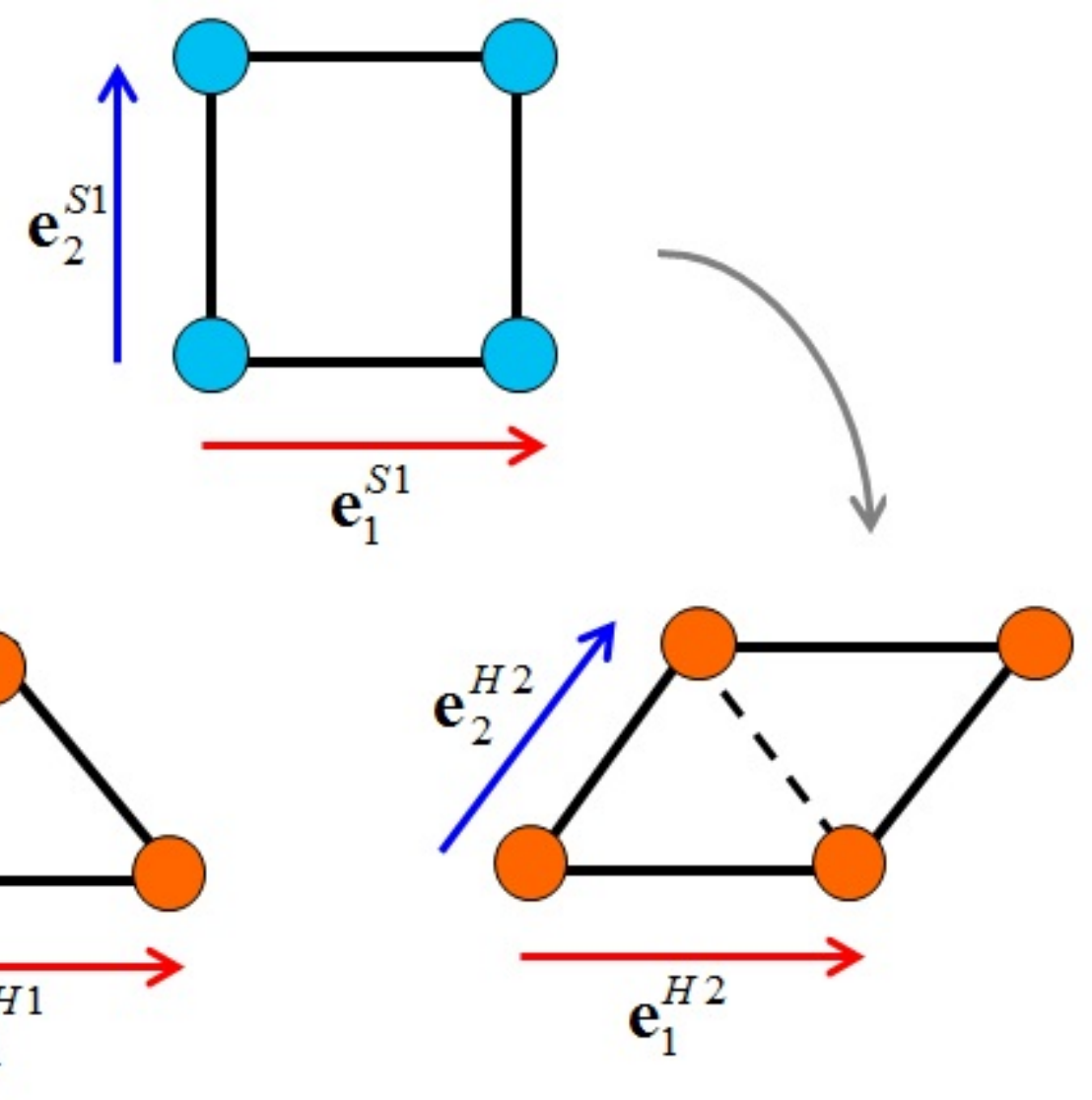

(c)

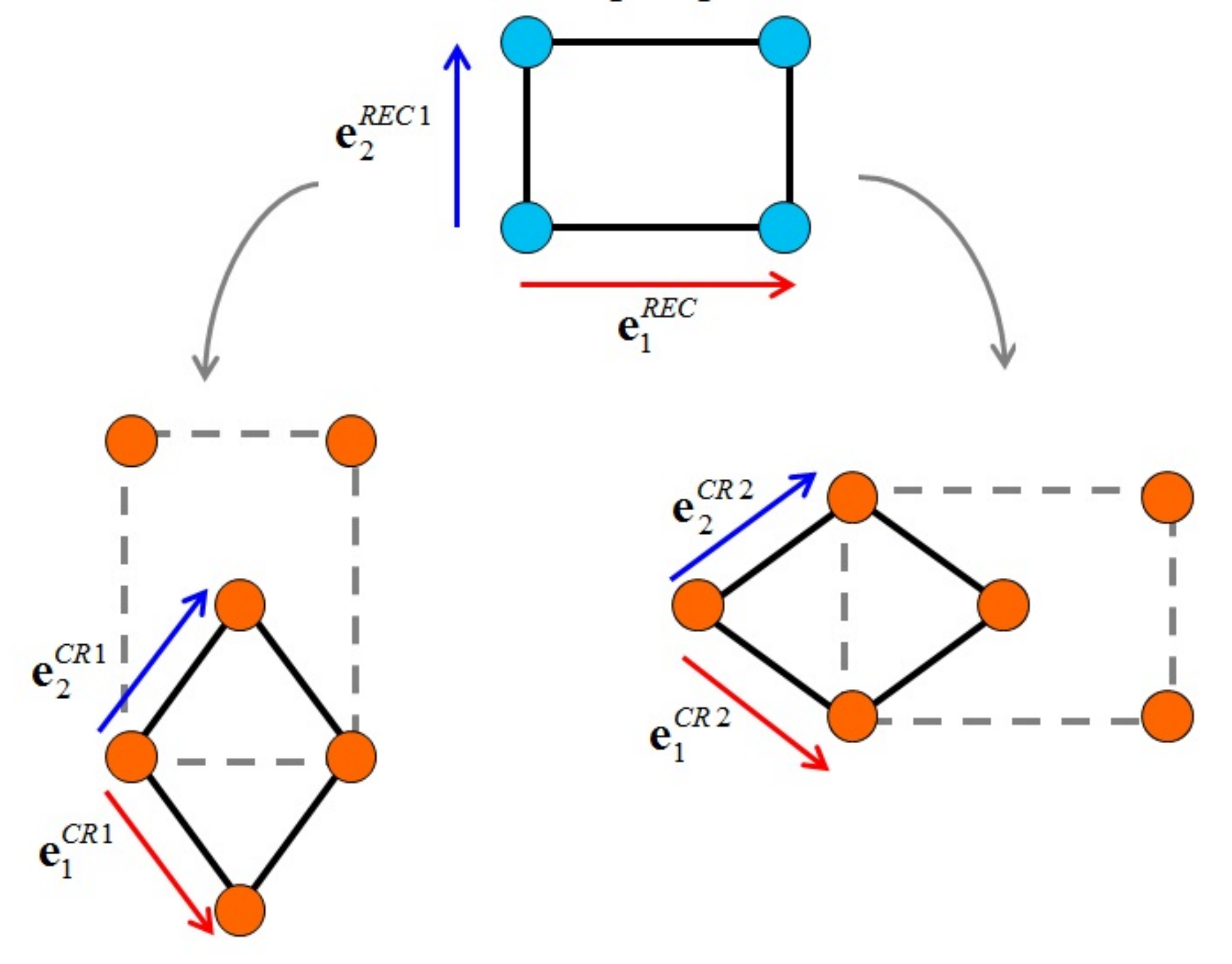

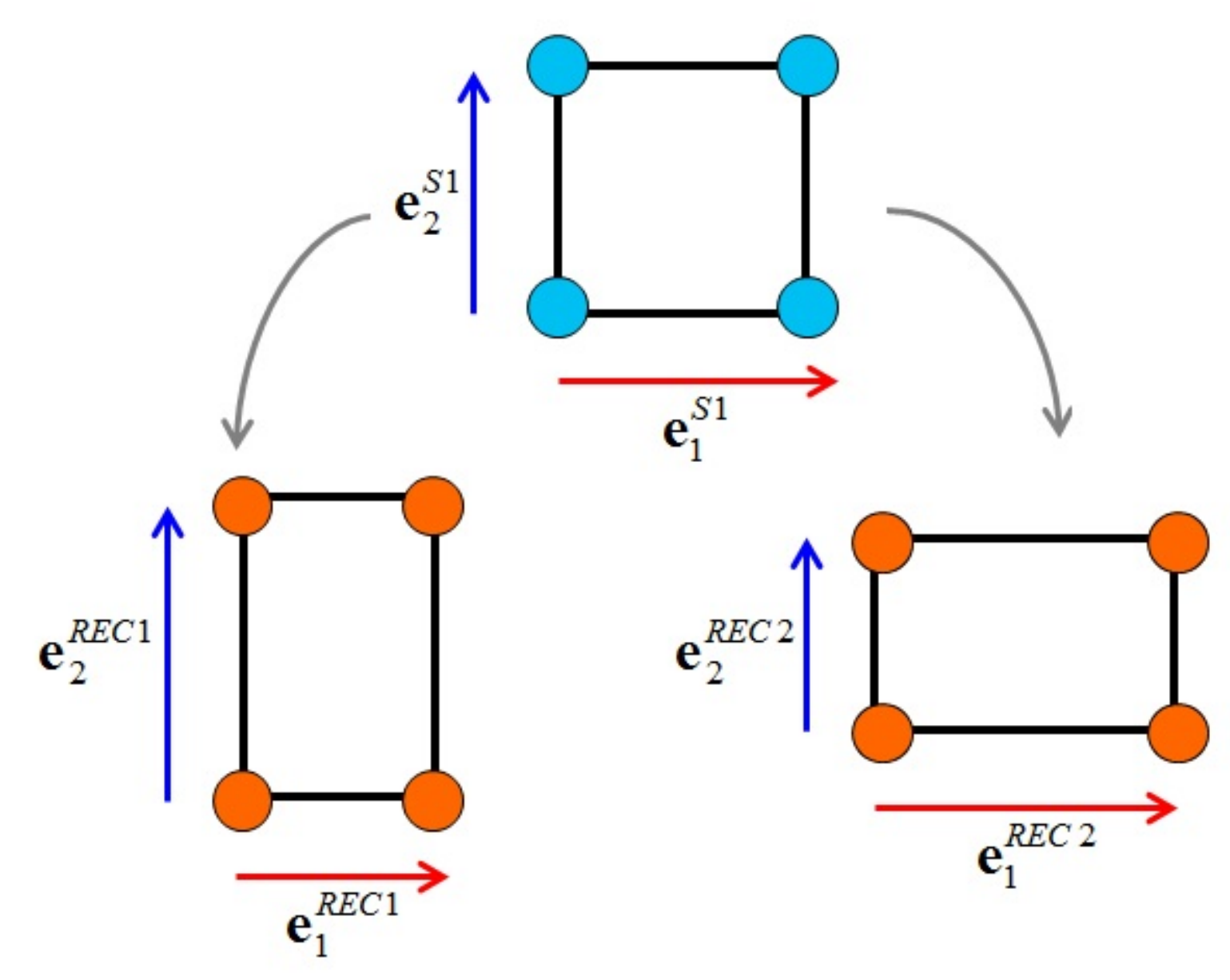


(a)

(b)
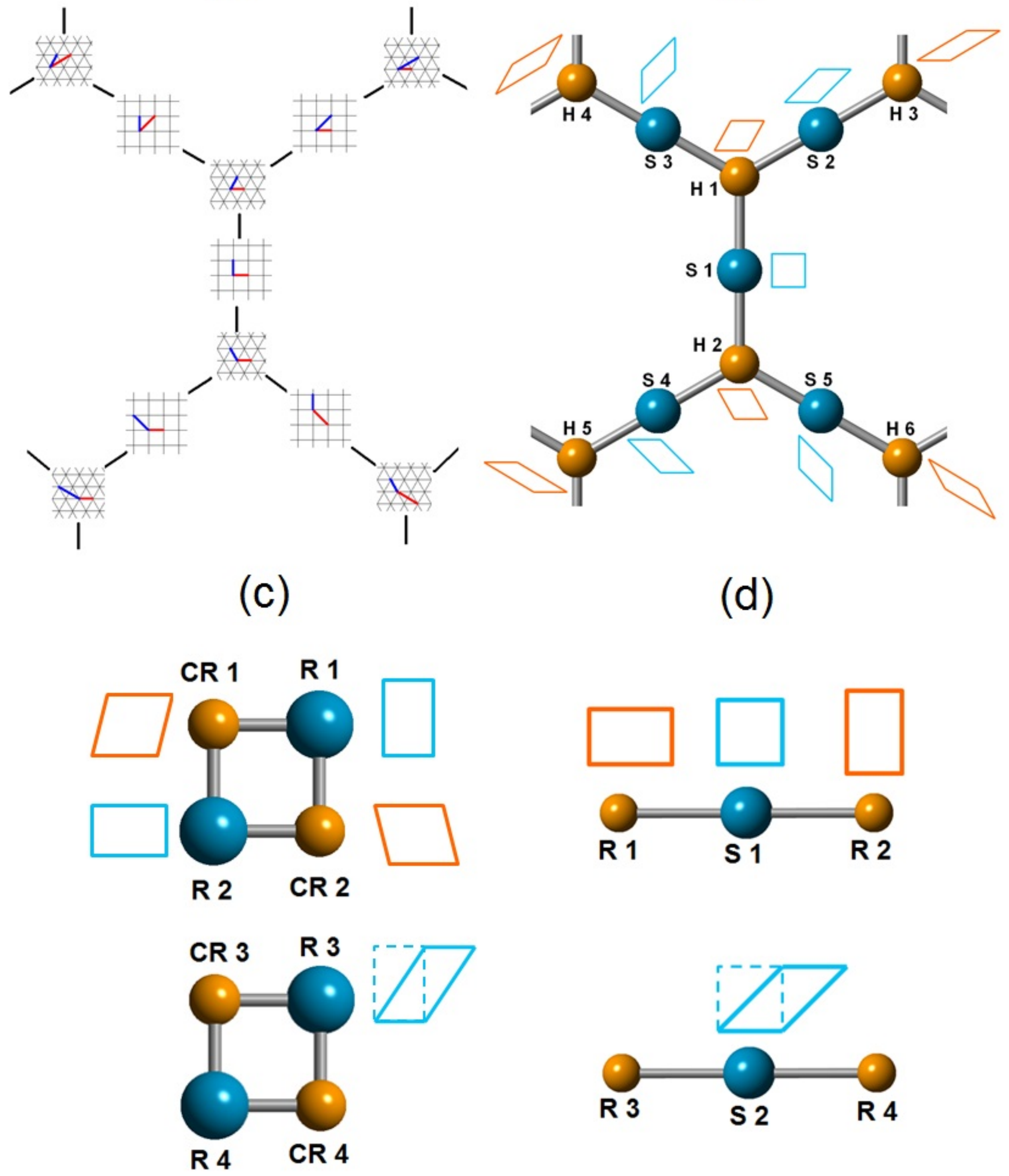
(a)

(b)

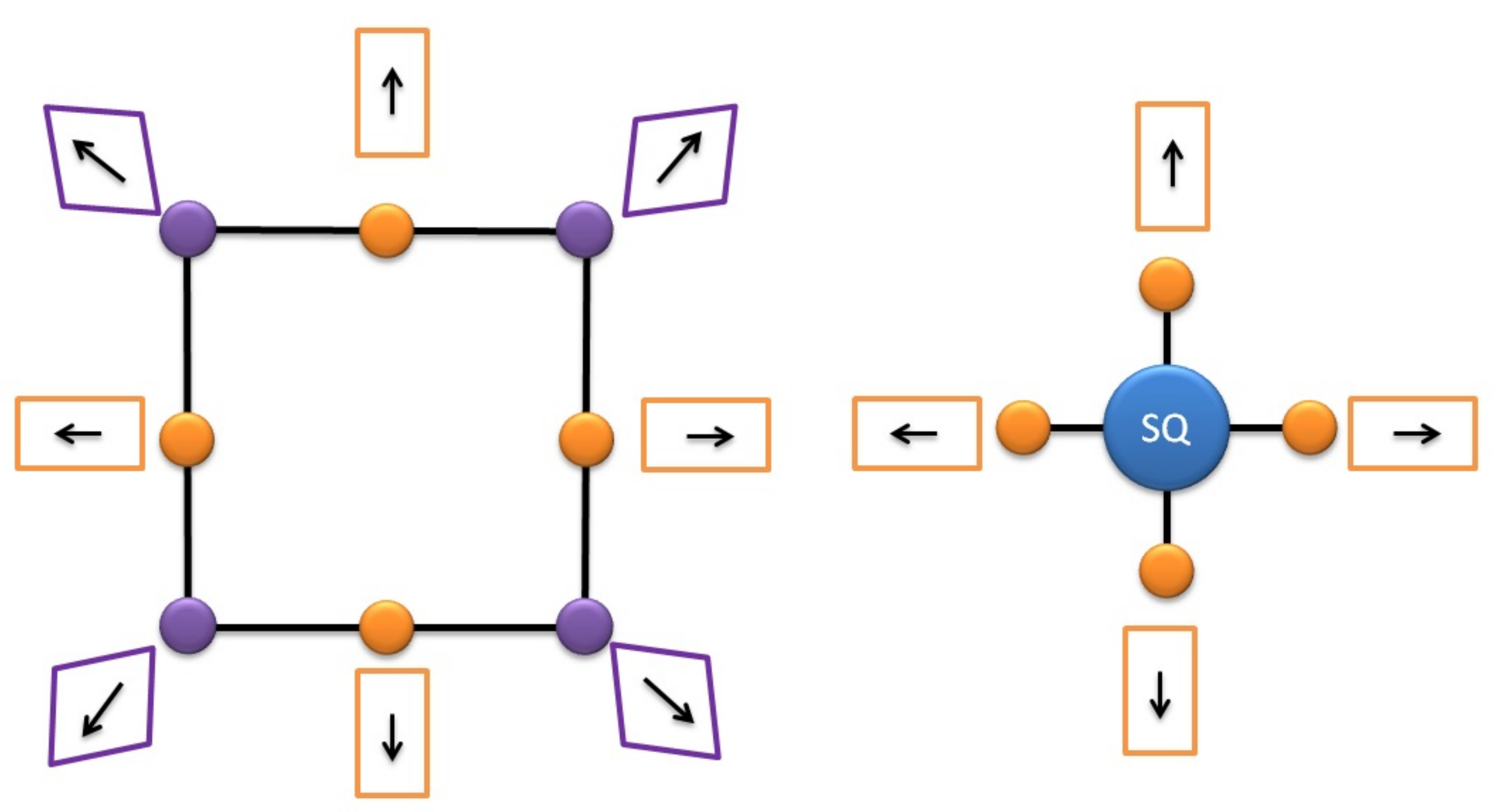

(c)

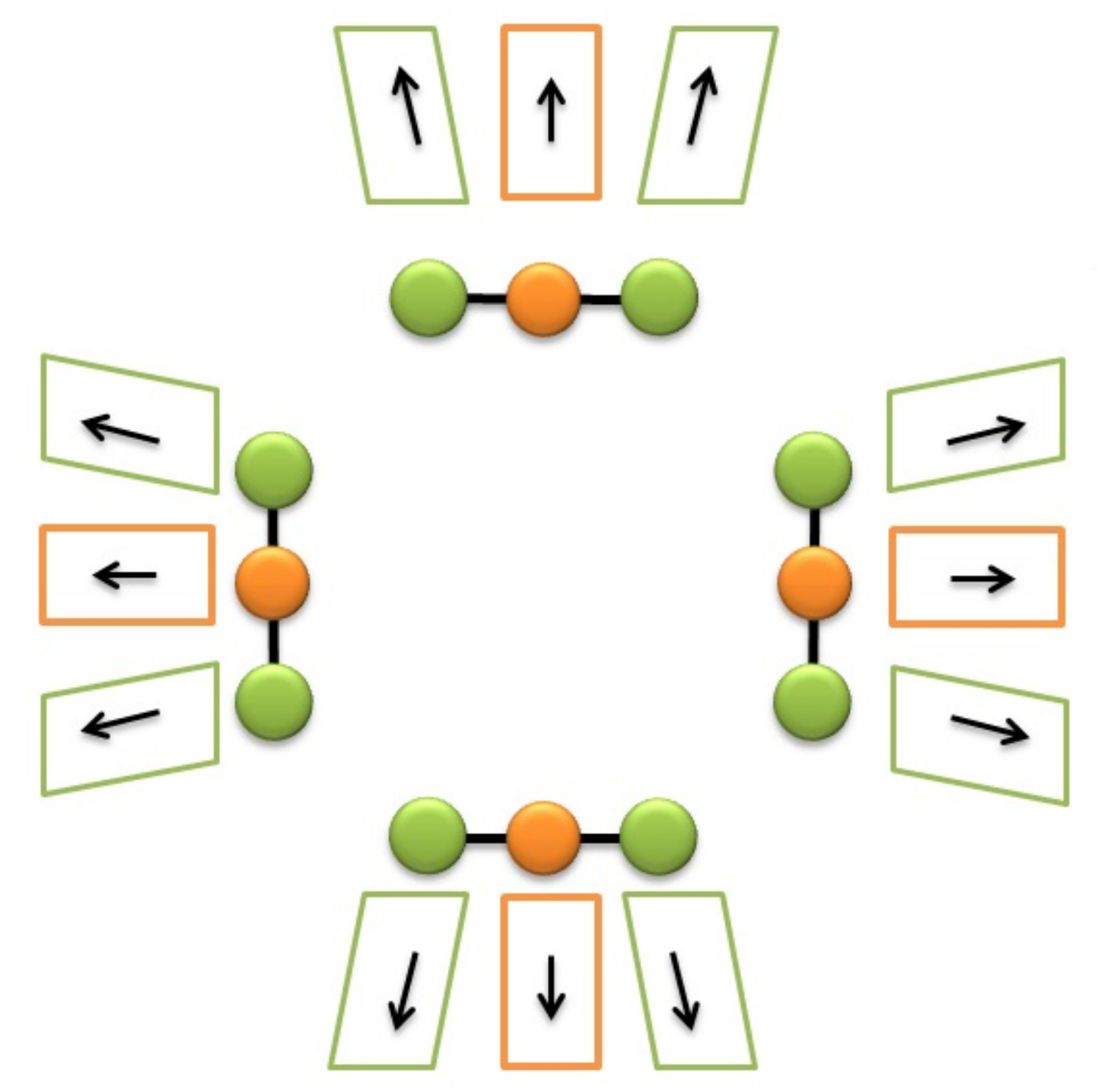

Square

Rectangle

Parallelogram

Centered Rectangle 


\begin{tabular}{|c|c|c|c|c|c|c|}
\hline Parent Phase & $\begin{array}{l}\text { Hexagonal } \\
(6.6 \mathrm{~mm})\end{array}$ & Trigonal & $\begin{array}{l}\text { Tetragonal } \\
\text { (4. } 4 \mathrm{~mm})\end{array}$ & Orth \& Mono & Triclinic & \multirow{7}{*}{$\begin{array}{l}\sqrt{ }: \text { Confirmed } \\
\sqrt{ } \text { : Newly discovered } \\
\boldsymbol{x} \text { : Impossible } \\
\text { ? : To be determined }\end{array}$} \\
\hline Product Phase & & & & & & \\
\hline $\begin{array}{c}\text { Hexagonal } \\
(6,6 \mathrm{~mm})\end{array}$ & ? & ? & $x$ & $?$ & $x$ & \\
\hline $\begin{array}{l}\text { Trigonal } \\
(3,3 \mathrm{~m})\end{array}$ & $?$ & ? & $\checkmark$ & $\checkmark$ & $x$ & \\
\hline $\begin{array}{c}\text { Tetragonal } \\
(4,4 \mathrm{~mm})\end{array}$ & $x$ & $\checkmark$ & ? & ? & $x$ & \\
\hline $\begin{array}{l}\text { Orth \& Mono } \\
(2, \mathrm{~m}, \mathrm{~mm} 2)\end{array}$ & $?$ & $\checkmark$ & $?$ & $?$ & $x$ & \\
\hline $\begin{array}{l}\text { Triclinic } \\
\text { (1) }\end{array}$ & $x$ & $x$ & $x$ & $x$ & $x$ & \\
\hline
\end{tabular}




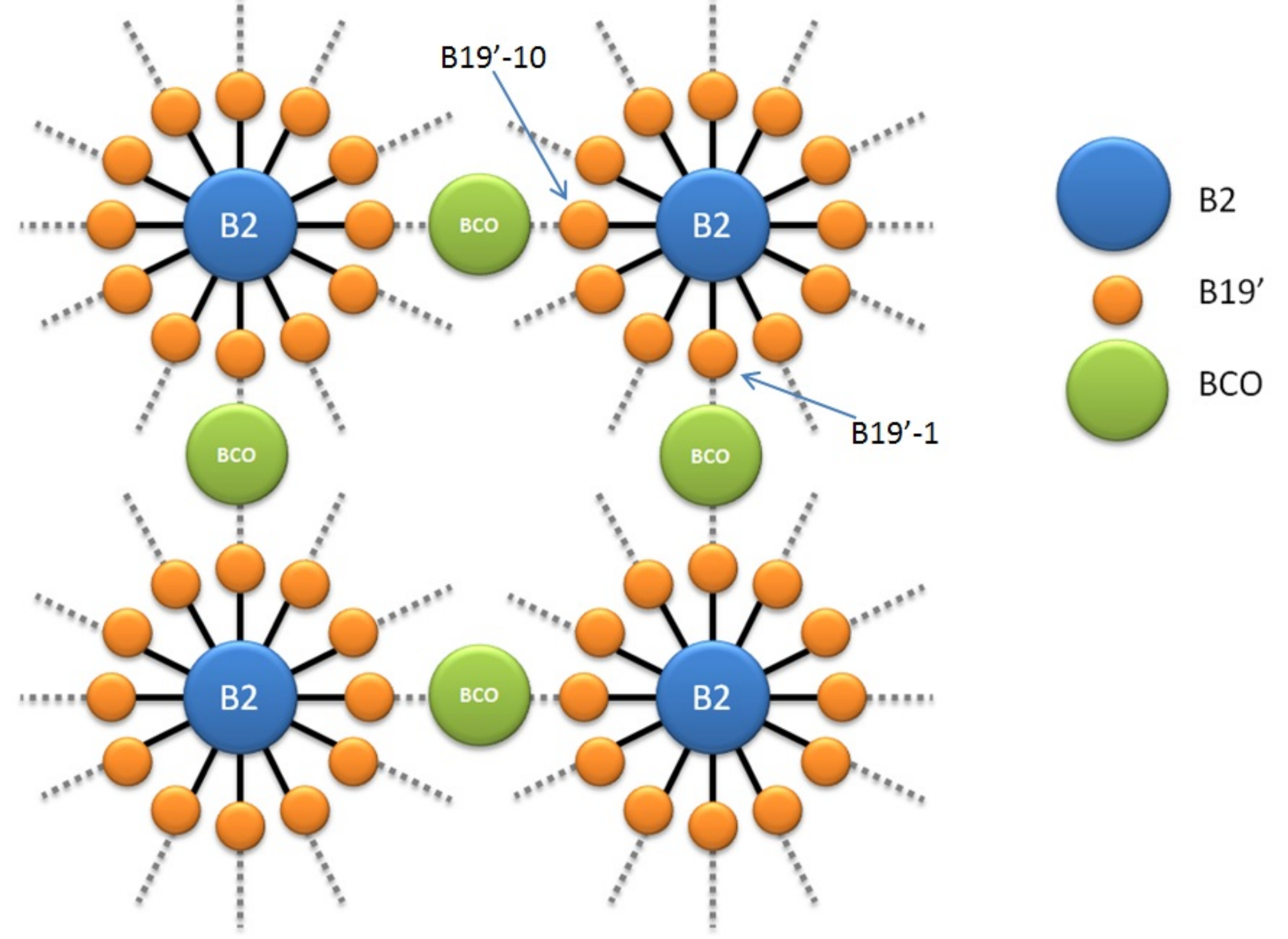




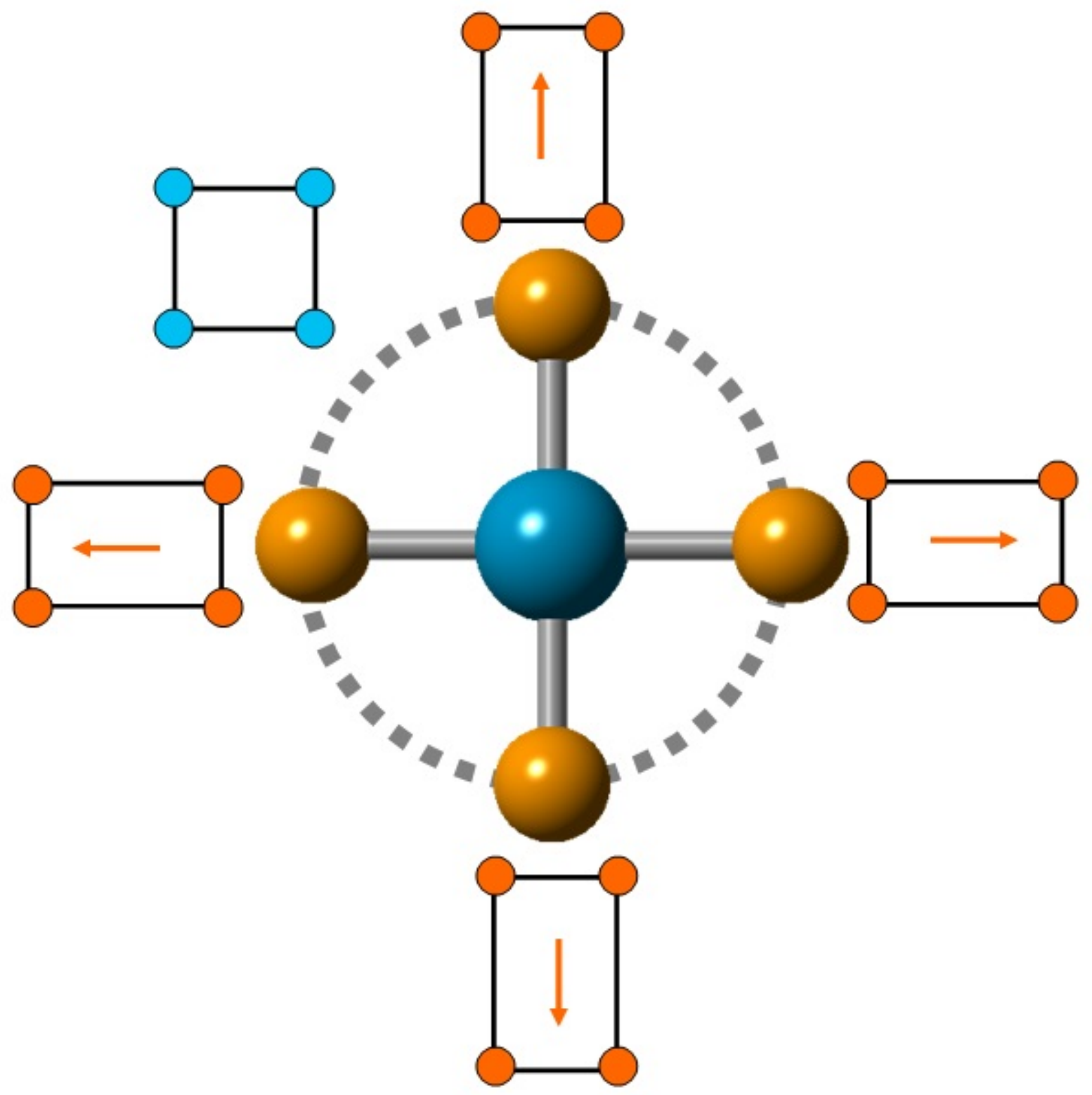




$$
\sum_{0}^{20} a_{0}^{20}
$$




\section{(a)}

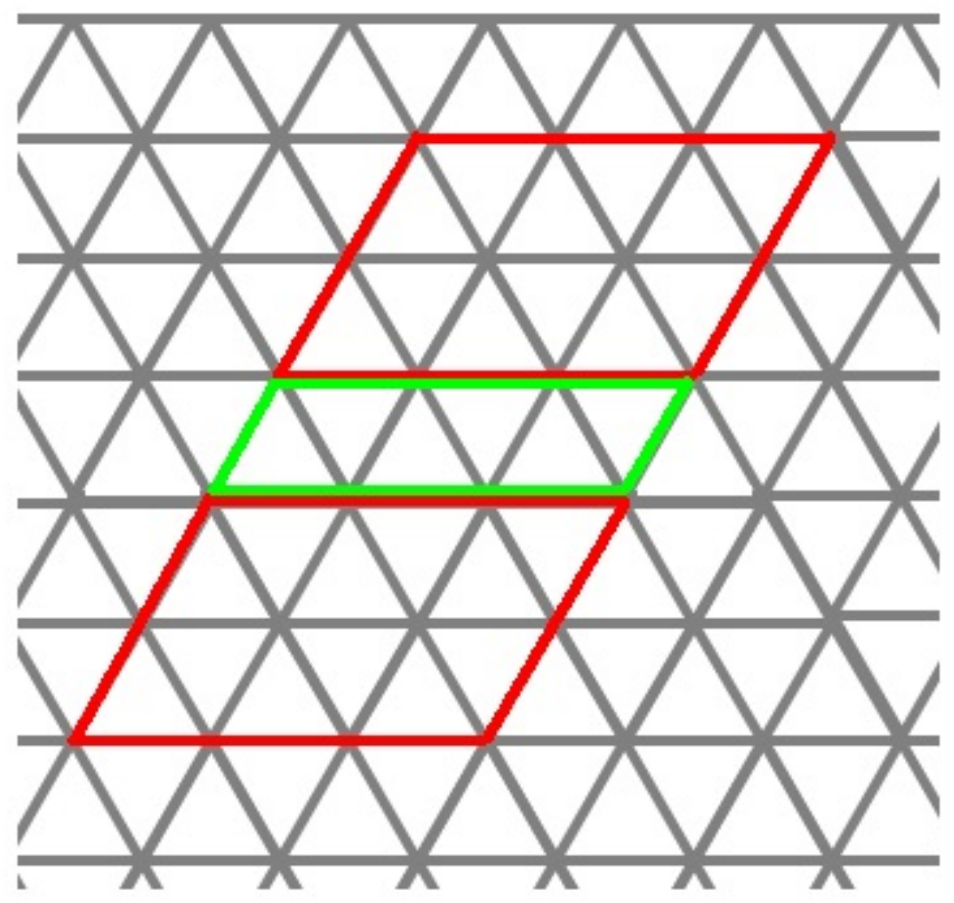

(b)

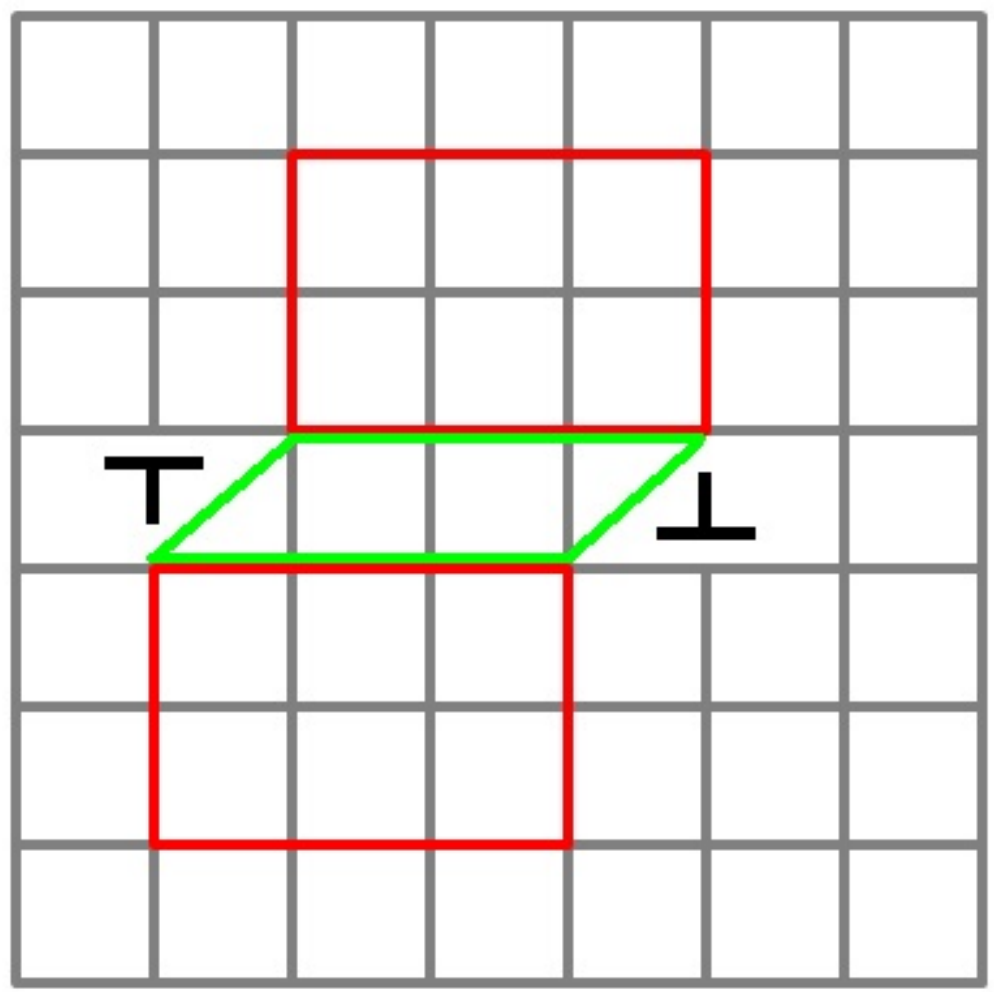




\section{(a)}

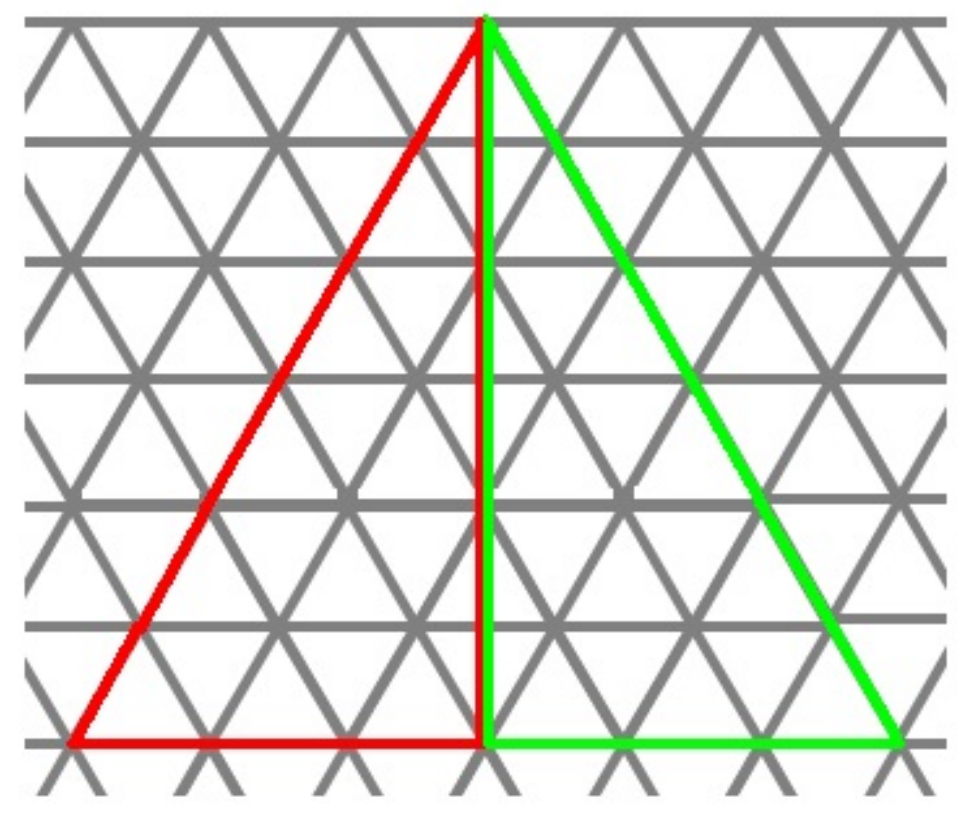

(b)

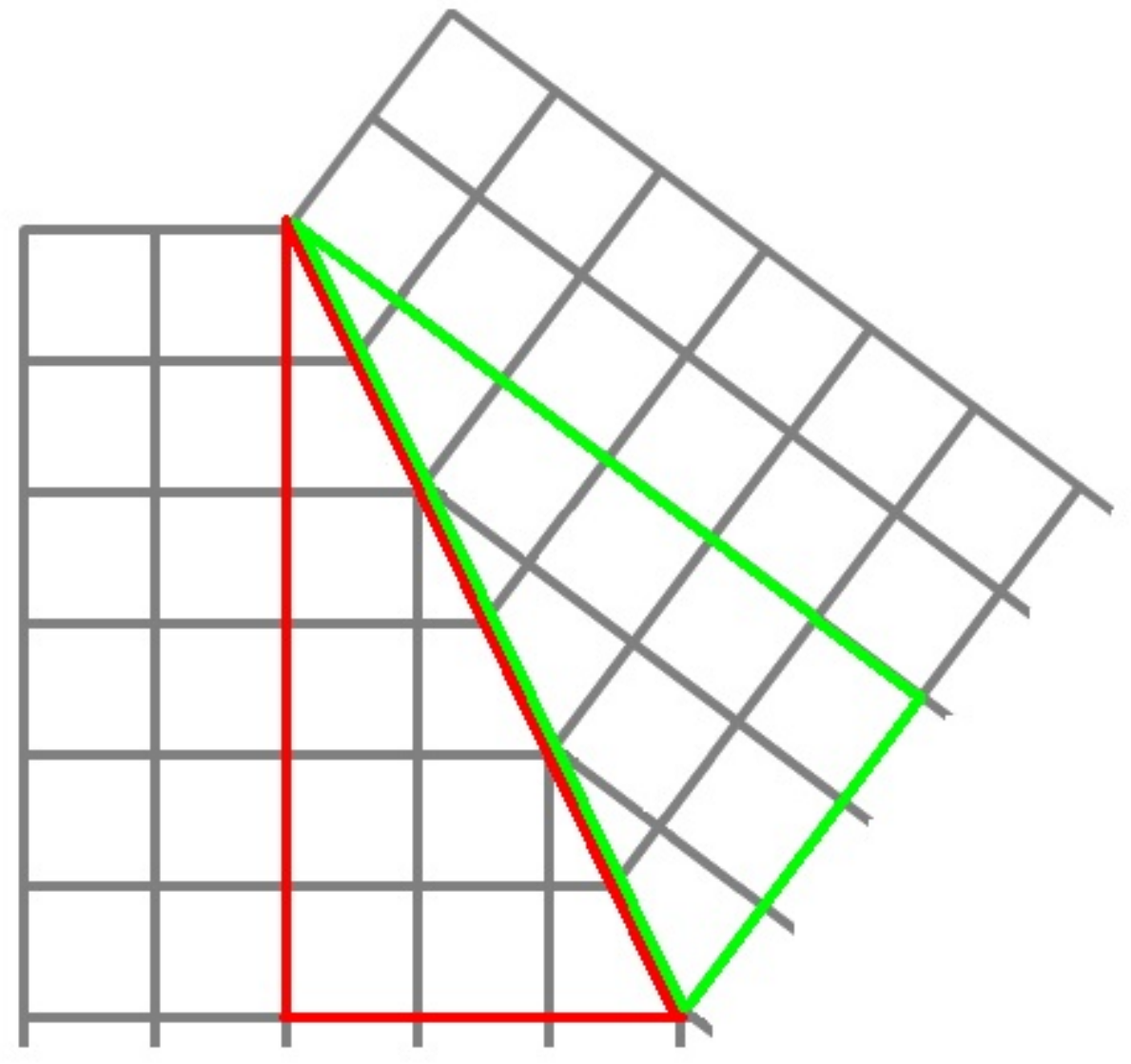


(a)

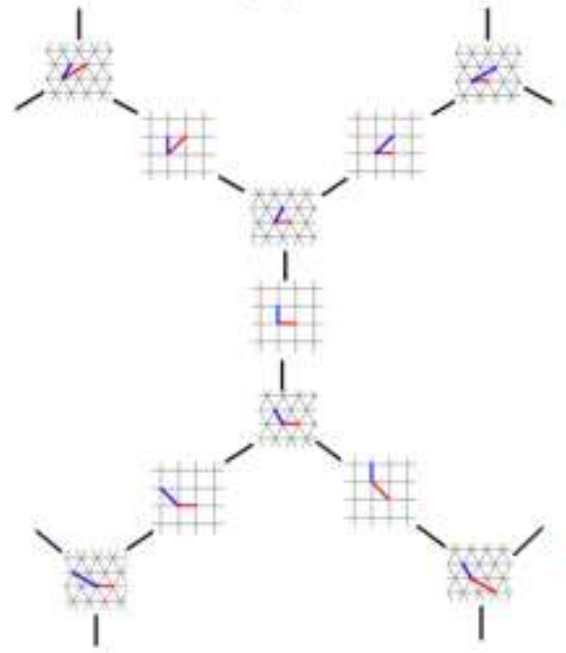

(c)
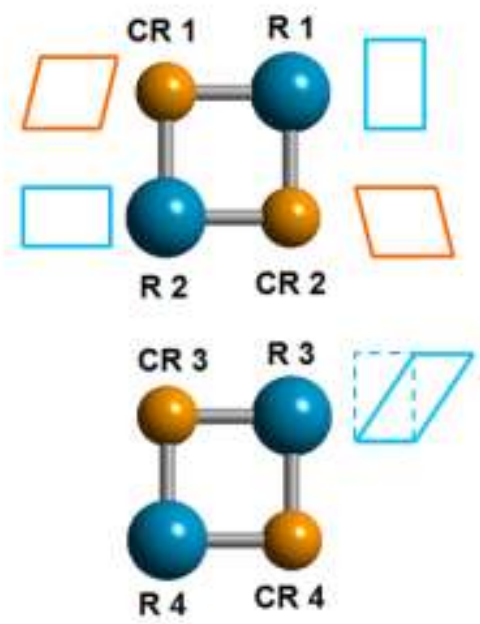

(b)
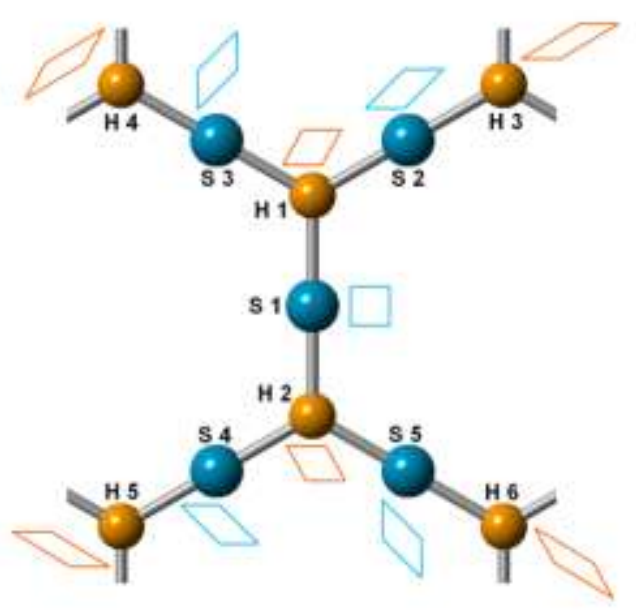

(d)
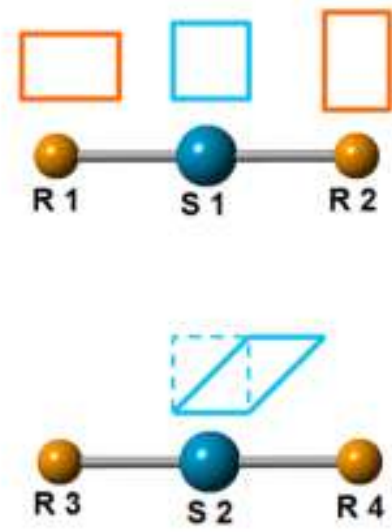

Construction of Phase Transition Graph (PTG) in 2D: (a) structural states (b) PTG for the square to hexagonal transition; (c) PTG for the rectangle to centered-rectangle transition; (d) PTG for the square to rectangle transition. 